\title{
Topology optimization of load-bearing capacity
}

\author{
Leyla Mourad · Jeremy Bleyer • Romain Mesnil • Joanna Nseir • \\ Karam Sab · Wassim Raphael
}

Received: date / Accepted: date

\begin{abstract}
The present work addresses the problem of maximizing a structure load-bearing capacity subject to given material strength properties and a material volume constraint. This problem can be viewed as an extension to limit analysis problems which consist in finding the maximum load capacity for a fixed geometry. We show that it is also closely linked to the problem of minimizing the total volume under the constraint of carrying a fixed loading. Formulating these topology optimization problems using a continuous field representing a fictitious material density yields convex optimization problems which can be solved efficiently using stateof-the-art solvers used for limit analysis problems. We further analyze these problems by discussing the choice of the material strength criterion, especially when considering materials with asymmetric tensile/compressive strengths. In particular, we advocate the use of a $L_{1}$ Rankine criterion which tends to promote uniaxial stress fields as in truss-like structures. We show that the considered problem is equivalent to a constrained Michell truss problem. Finally, following the idea of the SIMP method, the obtained continuous topology is post-processed by an iterative procedure penalizing intermediate densities. Benchmark examples are first considered to illustrate the method overall efficiency while
\end{abstract}

L. Mourad · J. Bleyer (凶) · R. Mesnil · K. Sab

Laboratoire Navier, Ecole des Ponts ParisTech, Univ Gustave Eiffel, CNRS

6-8 av. Blaise Pascal, Cité Descartes

77455 Champs-sur-Marne, France

Tel : +33 (0)164153743

E-mail: jeremy.bleyer@enpc.fr

L. Mourad · J. Nseir · W. Raphael

Université Saint Joseph, Faculté des sciences, Mar Roukos-

Dekwaneh, Lebanon final examples focus more particularly on no-tension materials, illustrating how the method is able to reproduce known structural patterns of masonry-like structures. This paper is accompanied by a Python package based on the FEniCS finite-element software library.

Keywords Topology optimization · Limit Analysis · Bearing capacity · Second-order cone programming · No-tension material · Michell truss

\section{Funding Information}

This work is part of the PhD thesis of L. Mourad who is supported by Université Paris-Est and Université Saint-Joseph.

\section{Introduction}

Construction materials used for primary structures of building and infrastructures are responsible for a consequent share of mankind's greenhouse gases emissions 26]. The reduction of weight through structural optimization is therefore a challenge of growing importance, even in the construction industry. Building structures have to comply on limit state design, which requires satisfying two principal criteria: Ultimate Limit State (ULS) and Serviceability Limit State (SLS). The two limit states differ fundamentally in their nature, since SLS criteria usually restrict the structures to perform in the elastic range, whereas ULS requires to check the structure maximal load-bearing capacity with respect to material non-linearities. When designing a structure with respect to ULS, it is important to take into account stress redistributions occurring during the material non-linearity phase. 
This non-linearity is particularly stringent for usual construction materials, like plain concrete which has different compressive and tensile strength.

Topology optimization is a mathematical problem of optimal material distribution in a given domain. In structural mechanics, topology optimization problems can be classified as stiffness optimization (related to SLS) and strength optimization (related to ULS). The seminal work of Michell 34, which dealt with fully-stressed continuous truss structures was in fact a compliance optimization. Michell truss have been extended in the middle of the twentieth century, either to construct discrete solutions [38, or to consider optimal plastic design of truss structures [39]. Bendsøe and Kikuchi [7] were among the first to introduce layout optimization where the distribution of the material within a domain is optimized rather that the shape of the domain itself.

Stiffness-based optimization is now a wellestablished field of research, with numerous applications in different industries [8]. Optimization problems aiming at maximizing stiffness are classically formulated as compliance minimization problems over displacement and stress fields satisfying the equations of linear elasticity (with a density-dependent elasticity tensor $\mathbf{C}(\rho)$ ), while the volume is bounded by a fraction of the total volume.

The solutions to such elastic compliance minimization problems result in densities continuously distributed between 0 and 1, which are hard to manufacture. Existing methods attempt at penalizing intermediate densities towards a black and white design and differ in the expression of the stiffness tensor $\mathbf{C}(\rho)$ [45. Homogenization methods make an analogy with perforated composite materials and replace the layout optimization by a sizing problem of the effective properties of this homogenized material [2]. This technique has been envisioned from the pioneering work of [7] and used to prove existence of solution on this relaxed problem. They are also compatible with penalized problem formulations that converge towards black and white designs 11. The SIMP Method (Solid Isotropic Material with Penalization) 8] uses a fictitious power law $\mathbf{C}(\rho)=\mathbf{C}_{0} \cdot \rho^{p}$. Although yielding non-convex problems for $p \geq 1$, heuristic iterative method and careful choice of power $p$ allow to quickly converge towards near-optimal black and white designs. The non-convexity implies non-uniqueness of solutions, which makes the penalized optimization problem sensitive to initialization, as well as numerical scheme e.g. the Method of Moving Asymptotes 48 or Optimal- ity Criterion [43] which may thus yield different results.

Stress-based optimization can be formulated by analogy with classical compliance minimization problems by considering a stress density cost function $J(\rho)=\int_{\mathcal{D}} \sigma_{\text {eff }} \mathrm{dx}$ where $\sigma_{\text {eff }}$ is an effective stress (e.g. the von Mises equivalent stress). The main advantage of such a formulation is that classical methods, e.g. SIMP or level-set methods 3] can readily be used. For that reason, this formulation has been used in several applications. Pedersen showed that the result of stress optimization and compliance optimization will differ if the strain energy is not consistent with the effective stress measure 36]. One of the main difficulties encountered in stress optimization is the difficulty to include many local stress constraints in the optimization problem as well as the existence of a singularity problem at zero densities 20].

Strictly speaking, these methods do not however optimize for load-bearing capacity (ULS design), as they typically consider stress fields which are solution of an elasticity problem. Finally, some works included fully non-linear elasto-plastic computations inside a topology optimization procedure [4, 5, 32, 49, 51, resulting in extremely high computational cost. The present work aims thus at exploring another formulation that optimize with respect to ULS, using yield design theory.

Yield design theory 40, 41 has been used in many areas of civil engineering to design structures based on the sole compatibility between the notions of equilibrium on one hand and resistance on the other hand. Typical fields of application include geotechnical problems (soil slope stability, footing bearing capacity) [18, design of reinforced-concrete structures [17, 35], especially using strut-and-tie methods 42 or rigid-block stone or masonry structures, such as Heyman's works [23, 24] assuming infinite compressive strength and zero tensile strength for the material.

Yield design theory can be conceived as the extension of classical limit analysis theorems 25] in the case of perfect plasticity which provide lower and upper bound approaches bracketing the structure ultimate load. The former relies for instance on finding a stress field which should be statically admissible with a given loading and verify the local material strength criterion. Yield design/limit analysis approaches can now be efficiently solved numerically by formulating them as convex (more precisely conic) optimization problems 12, 29, 31, 46, 50, and solved using dedicated solvers.

A noteworthy implementation of the lower bound theorem for the design of masonry structures was introduced with thrust network analysis (TNA), which was 
presented by Block and Ochsendorf [13, 14]. TNA essentially relies on the computation of statically admissible membrane force fields satisfying Heyman's hypotheses using the force-density method. In recent studies, the Block Research Group from ETH Zürich has worked over constructing dry-stack no-tension structures to show that those structures are capable of putting in place a complex shape that surpasses beyond simple walls, or traditional vault geometries [15, 33.

Only few works concentrated on optimization with respect to the structure ultimate limit state. One can mention the work of Damkilde and Krenk [19] which determined an optimized material distribution in reinforced concrete slabs in bending. More recently, similar ideas have been used in 21, 22, 27, to propose strength-based topology optimization of von Mises plastic materials using limit analysis formulations. Our contribution aims at providing a general formulation of topology optimization in such a context by relying on the concepts of yield design/limit analysis theory and on convex optimization tools.

The manuscript is organized as follows: section 2 formulates the problem of maximizing the structure load bearing capacity for a predefined volume constraint. The problem is initially formulated as a mixed-integer programming and parallels with a volume-minimization problem are drawn. Section 3 present both problem relaxations using a continuous pseudo-density field. The properties of the obtained convex problems are then detailed. Section 4 presents usual material strength criteria in civil engineering, especially regarding tension/ compression asymmetric materials. Section 5 presents the discretization and resolution procedure for solving the convex problems. A penalization procedure is also proposed. Finally, the efficiency of the proposed formulation is illustrated in section 6 through various examples.

\section{Maximizing the load-bearing capacity of a structure}

\subsection{A brief review of limit analysis theory}

In this section, we recall the general concepts of limit analysis (or yield design) theory for structures with a known geometry $\Omega$. When considering perfectly plastic materials, the limit analysis theory provides a direct characterization of the structure load-bearing capacity corresponding to global plastic collapse. Note that the load-bearing capacity differs from the elastic limit of a structure (obtained when considering strength constraints for an elastic solution) because of the struc- ture's ability to redistribute loads when its constitutive materials offer sufficient ductility. As a result, the stress field associated with the structure collapse may be quite different from the elastic solution. It is therefore expected that optimized design obtained from stress fields at incipient collapse may be quite different from optimized design obtained in the elastic regime.

The load-bearing capacity is obtained by finding the maximum load amplification factor $\lambda$ for which there exists an internal stress field $\boldsymbol{\sigma}$ which can balance the loading and still comply with a strength criterion $\sigma \in G$ at every point in $\Omega$ where $G$ is a convex set containing 0 . More precisely, the limit load $\Lambda^{+}$can be found as the solution to the following convex optimization problem:

$$
\begin{aligned}
& \Lambda^{+}=\max _{\lambda, \boldsymbol{\sigma}} \lambda \\
& \text { s.t. } \operatorname{div} \boldsymbol{\sigma}+\boldsymbol{f}=0 \text { in } \Omega \\
& \boldsymbol{\sigma} \cdot \boldsymbol{n}=\lambda \boldsymbol{T} \quad \text { on } \partial \Omega_{T} \\
& \boldsymbol{\sigma} \in G \quad \text { in } \Omega
\end{aligned}
$$

in which we considered the body force $f$ to be fixed whereas we look for the maximal value of the reference surface tractions $\boldsymbol{T}$ acting on some part $\partial \Omega_{T}$ of the boundary. Also note that the local balance equation $\operatorname{div} \boldsymbol{\sigma}+\boldsymbol{f}=0$ is to be understood in the sense of distributions i.e. $\boldsymbol{\sigma} \cdot \boldsymbol{n}$ must be continuous.

As regards numerical resolution of problem (1), it turns out that many usual strength criteria can be formulated using second-order cone constraints [9, 31]. When combined with dedicated finite element discretization, the discrete counterpart of (1) falls into the class of second-order cone programs (SOCP) [30. Such convex optimization problems are particularly important because they can be solved efficiently using interior-point algorithms implemented in dedicated solvers such as Mosek, CPLEX, etc.

\subsection{Extending limit analysis to topology optimization}

Building upon the concepts of limit analysis, we now aim at finding an optimized structure $\Omega \subseteq \mathcal{D}$ contained in a computational domain $\mathcal{D}$ with maximum load-bearing capacity for a given material volume fraction constraint $\eta$. Problem (1) written on the unknown geometry $\Omega$ can be re-expressed on the computational domain $\mathcal{D}$ by introducing an additional optimization variable in the form of a characteristic func- 
tion $\rho(\boldsymbol{x}) \in\{0,1\}$ with $\rho(\boldsymbol{x})=1$ when $\boldsymbol{x} \in \Omega$. Such a problem reads as:

$$
\begin{aligned}
\lambda^{+}=\max _{\lambda, \boldsymbol{\sigma}, \rho} \lambda & \\
\text { s.t. } & \operatorname{div} \boldsymbol{\sigma}+\rho \boldsymbol{f}=0 \text { in } \mathcal{D} \\
& \boldsymbol{\sigma} \cdot \boldsymbol{n}=\lambda \boldsymbol{T} \quad \text { on } \partial \mathcal{D}_{T} \\
& \boldsymbol{\sigma} \in \rho G \quad \text { in } \mathcal{D} \\
& \int_{\mathcal{D}} \rho \mathrm{dx} \leq \eta|\mathcal{D}| \\
& \rho \in\{0,1\}
\end{aligned}
$$

in which $|\mathcal{D}|$ denotes the volume of $\mathcal{D}$ and $\partial \mathcal{D}_{T}$ the boundary part on which tensile loads are applied. In the above problem, the main difference with respect to (1) is that the strength domain $G$ is replaced by an homothetic domain $\rho G$. In particular, $\boldsymbol{\sigma}(\boldsymbol{x}) \in \rho(\boldsymbol{x}) G$ enforces that $\boldsymbol{\sigma}(\boldsymbol{x}) \in G$ for $\boldsymbol{x} \in \Omega$ (i.e. where $\rho(\boldsymbol{x})=1$ ) and $\boldsymbol{\sigma}(\boldsymbol{x})=0$ for $\boldsymbol{x} \notin \Omega$ (i.e. where $\rho(\boldsymbol{x})=0$ ), provided that $G$ is bounded. As a result, if $\rho$ is known, (2) is indeed equivalent to $(1)$ formulated on $\Omega$.

\subsection{A related volume-minimization problem}

Instead of maximizing the load-bearing capacity for a given volume fraction constraint as in (2), one can also attempt at minimizing the total material volume under the condition that the structure can sustain (according to limit analysis theory) a given load level $\lambda \boldsymbol{T}$, with $\lambda$ now having a prescribed value. Such a volumeminimization problem has already been considered in previous works [22, 27] and reads:

$$
\begin{aligned}
& \eta^{-}=\min _{\boldsymbol{\sigma}, \rho} \frac{1}{|\mathcal{D}|} \int_{\mathcal{D}} \rho \mathrm{dx} \\
& \text { s.t. } \operatorname{div} \boldsymbol{\sigma}+\rho \boldsymbol{f}=0 \text { in } \mathcal{D} \\
& \boldsymbol{\sigma} \cdot \boldsymbol{n}=\lambda \boldsymbol{T} \quad \text { on } \partial \mathcal{D}_{T} \\
& \boldsymbol{\sigma} \in \rho G \quad \text { in } \mathcal{D} \\
& \rho \in\{0,1\}
\end{aligned}
$$

The relation between (2) and (3) will be further explored in the subsequent section when considering their convex relaxations.

\section{Convex continuous relaxation}

\subsection{Relaxed problems definitions}

Both problems (2) and (3) are extremely difficult to solve in practice due to the binary constraint $\rho \in\{0,1\}$.
Following the classical procedure of topology optimization, both problems are relaxed by considering instead a continuous constraint $\rho \in[0 ; 1]$ :

$$
\begin{aligned}
\lambda^{+}=\max _{\lambda, \boldsymbol{\sigma}, \rho} & \lambda \\
\text { s.t. } & \operatorname{div} \boldsymbol{\sigma}+\rho \boldsymbol{f}=0 \text { in } \mathcal{D} \\
& \boldsymbol{\sigma} \cdot \boldsymbol{n}=\lambda \boldsymbol{T} \quad \text { on } \partial \mathcal{D}_{T} \\
& \boldsymbol{\sigma} \in \rho G \quad \text { in } \mathcal{D} \\
& \int_{\mathcal{D}} \rho \mathrm{dx} \leq \eta|\mathcal{D}| \\
& 0 \leq \rho \leq 1
\end{aligned}
$$

and

$$
\begin{aligned}
& \eta^{-}=\min _{\boldsymbol{\sigma}, \rho} \frac{1}{|\mathcal{D}|} \int_{\mathcal{D}} \rho \mathrm{dx} \\
& \text { s.t. } \operatorname{div} \boldsymbol{\sigma}+\rho \boldsymbol{f}=0 \text { in } \mathcal{D} \\
& \boldsymbol{\sigma} \cdot \boldsymbol{n}=\lambda \boldsymbol{T} \quad \text { on } \partial \mathcal{D}_{T} \\
& \boldsymbol{\sigma} \in \rho G \quad \text { in } \mathcal{D} \\
& 0 \leq \rho \leq 1
\end{aligned}
$$

It turns out that both relaxed problems LOAD-MAX and (VOL-MIN) are convex problems. Indeed, all constraints are linear except for the strength constraint $\boldsymbol{\sigma} \in \rho G$ which is convex (see proof in Appendix A). This property motivates the use of the homothetic scaling for the density-dependent strength domain $\boldsymbol{\sigma} \in G(\rho)=\rho G$. Indeed, it enables to enforce $\boldsymbol{\sigma}=0$ and $\boldsymbol{\sigma} \in G$ in the two limit cases $\rho=0$ and $\rho=1$ respectively while the relaxed problem becomes convex with respect to the pair $(\boldsymbol{\sigma}, \rho)$ of optimization variables, a property which will be efficiently taken advantage of in section 5 .

Finally, let us point out that we will always solve the above problems simultaneously for $\boldsymbol{\sigma}$ and $\rho$, in a monolithic fashion. Indeed, contrary to elastic-based topology optimization problems, solving the above problems for $\boldsymbol{\sigma}$ only, at fixed $\rho$, is a difficult problem, since it is, in fact, a limit analysis problem. The coupled problem therefore adds only an extra scalar optimization variable compared to a standard limit analysis computation and avoids the need of alternate minimization between the problem on $\boldsymbol{\sigma}$ and the problem on $\rho$.

\subsection{Properties of relaxed problems solutions}

In this paragraph and in Appendix $B$ we will consider the optimal objective value $\lambda^{+}(\eta)$ of problem LOAD-MAX as a function of the volume fraction parameter $\eta$ as well as the optimal objective value $\eta^{-}(\lambda)$ of problem (VOL-MIN as a function of the load factor parameter $\lambda$. One can show that both functions are 
non-decreasing functions with $\lambda^{+}(1)=\Lambda^{+}$being the solution to the limit analysis problem (1). Similarly, $\eta^{-}(\lambda)=+\infty$ for $\lambda>\Lambda^{+}$since (VOL-MIN) becomes infeasible in this case. Finally, one can also show that problems (LOAD-MAX) and (VOL-MIN) are in fact equivalent since $\lambda^{+}(\eta)$ and $\eta^{-}(\lambda)$ are inverses of each other, see Appendix B for the proof. See also Figure 3 for more details.

If we remove the upper bound constraint $\rho \leq 1$ in problems (LOAD-MAX) and (VOL-MIN), then we can easily show that $\lambda^{+}(\eta)=C \eta$ and $\eta^{-}(\lambda)=\frac{1}{C} \lambda$ with $C$ being a constant. This constant corresponds to $C=\left.\frac{d \lambda^{+}}{d \eta}\right|_{\eta=0}$ when considering the initial formulations including the upper bound constraint $\rho \leq 1$. Without the upper bound constraint, problems (LOAD-MAX) and VOL-MIN will therefore always give the same optimized density field $\rho$ up to a scaling factor, irrespective of the value of the design load level $\lambda$ or the maximum volume fraction $\eta$. In particular, both problems will always give a solution for any value of $\eta$ or $\lambda$.

\section{On the choice of the material strength criterion}

\subsection{Strength criterion for asymmetric materials}

Previous works on limit analysis-based volume minimization considered only either plane strain [27] or plane stress 22 von Mises strength criteria. In the present work, the formulation has been established for any strength criterion $G$ which can be chosen depending on the material under investigation.

For instance, most materials in civil engineering exhibit different strength properties in tension and compression. Incorporating such tension/compression asymmetry can be achieved using a Drucker-Prager, Mohr-Coulomb or, more simply, a Rankine strength criterion (see Figure 1). The latter can be expressed as:

$\boldsymbol{\sigma} \in G_{\text {Rankine }} \Longleftrightarrow-f_{c} \leq \sigma_{I}, \sigma_{I I}, \sigma_{I I I} \leq f_{t}$

where $\sigma_{J}$ with $J=I, I I, I I I$ denote the principal stresses and $f_{c}$ (resp. $f_{t}$ ) is the material compressive (tensile) strength. In 3D conditions, the Rankine crite-

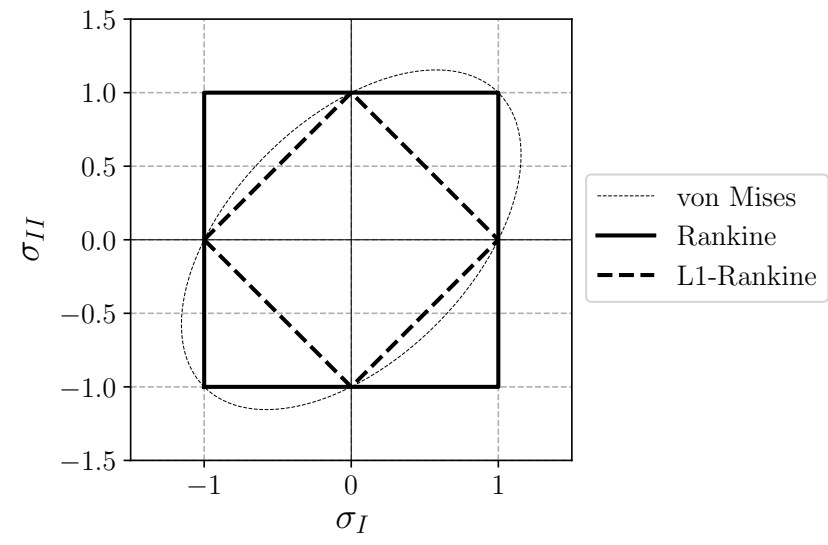

(a) Symmetric strengths $f_{t}=f_{c}=1$

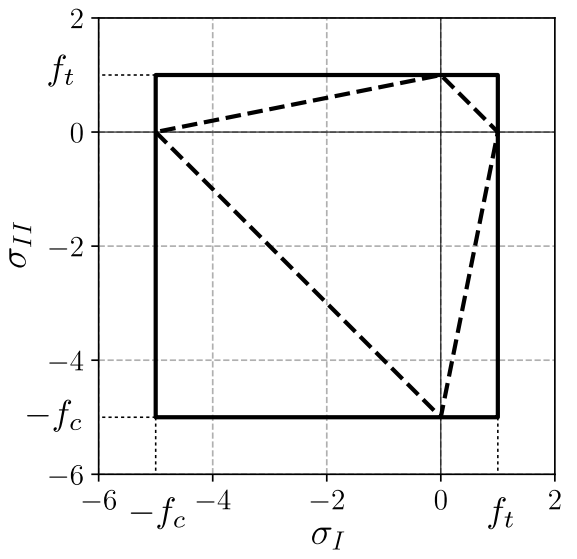

(b) Asymmetric strengths $f_{c}=5, f_{t}=1$

Fig. 1: Strength criteria shapes in the principal stress space

rion can be expressed using SDP constraints but for 2D conditions these can be reduced to SOCP constraints:

$$
\begin{aligned}
& \left(f_{t}-\sigma_{x x}\right)\left(f_{t}-\sigma_{y y}\right) \geq \sigma_{x y}^{2} \\
\sigma \in G_{\text {Rankine }} \Longleftrightarrow & \left(f_{c}+\sigma_{x x}\right)\left(f_{c}+\sigma_{y y}\right) \geq \sigma_{x y}^{2} \\
& -f_{c} \leq \sigma_{x x}, \sigma_{y y} \leq f_{t}
\end{aligned}
$$

4.2 A $L_{1}$-Rankine criterion and its link to truss-like designs

One objective of topology optimization amounts to finding optimized structures which often exhibit trusslike designs. Away from truss connections or supports, the stress state is uniaxial in the truss members so that $-f_{c} \leq \sigma_{I} \leq f_{t}$ and $\sigma_{I I}=0$. This condition is equivalent to saying that $\boldsymbol{\sigma}$ must be of rank 1 ( $L_{0}$ Schatten norm). Unfortunately, the induced set is non-convex. Similarly, 
the Rankine criterion can be viewed as a $L_{\infty}$-norm (spectral norm) on the principal stresses $\|\boldsymbol{\sigma}\|_{\infty} \leq f_{0}$ for similar tension and compression strengths $f_{c}=f_{t}=f_{0}$. Although this set is convex, numerical examples of section 6 will show that it has a tendency to promote biaxial stress states instead of uniaxial ones. A compromise between the sparsity-inducing $L_{0}$-norm and the convex $L_{\infty}$-norm is the nuclear $L_{1}$-norm: $\|\boldsymbol{\sigma}\|_{1}=\left|\sigma_{I}\right|+\left|\sigma_{I I}\right| \leq$ $f_{0}$. Indeed, the $L_{1}$-norm is the tightest convex relaxation norm of the $L_{0}$-norm and has been used in many applications for inducing sparse solutions [6] in compressed sensing or image processing applications. As a result, we advocate for the use of a $L_{1}$-Rankine criterion in order to promote sparse (i.e. as uniaxial as possible) principal stress states at the optimum. In the case of asymmetric tensile/compressive strengths this $L_{1}$-Rankine criterion reads as (see Figure 1 for a comparison of the criterion shapes in $2 \mathrm{D})$ :

$\boldsymbol{\sigma} \in G_{\text {L1-Rankine }} \Leftrightarrow \sum_{J=I, I I, I I I} \max \left\{-\frac{\sigma_{J}}{f_{c}} ; \frac{\sigma_{J}}{f_{t}}\right\} \leq 1$

We also refer to C for a second-order cone formulation of the $2 \mathrm{D} L_{1}$-Rankine criterion.

We further justify this choice by relating it to volume-optimal trusses studied by Michell [34. Continuous volume-optimal 2D trusses have indeed been characterized as finding a $2 \mathrm{D}$ stress state complying with equilibrium conditions and minimizing the quantity $\int_{\mathcal{D}}\left(\left|\sigma_{I}\right|+\left|\sigma_{I I}\right|\right) \mathrm{dx}$ in [2, 47]. We see that problem VOL-MIN with a symmetric $L_{1}$-Rankine criterion can be written as:

$$
\begin{aligned}
& \min _{\boldsymbol{\sigma}, \rho} \frac{1}{|\mathcal{D}|} \int_{\mathcal{D}} \rho \mathrm{dx} \\
& \text { s.t. equilibrium } \\
& \quad\left|\sigma_{I}\right|+\left|\sigma_{I I}\right| \leq \rho f_{0} \text { in } \mathcal{D}
\end{aligned}
$$

which is also equivalent to:

$$
\begin{aligned}
& \min _{\boldsymbol{\sigma}} \frac{1}{f_{0}|\mathcal{D}|} \int_{\mathcal{D}}\left(\left|\sigma_{I}\right|+\left|\sigma_{I I}\right|\right) \mathrm{dx} \\
& \text { s.t. equilibrium }
\end{aligned}
$$

which is exactly the characterization of volume-optimal continuous 2D trusses discussed in [28, 47]. A similar equivalence can be obtained for asymmetric strengths.

Note that in the above problems, we removed the upper bound condition $\rho \leq 1$ (see the discussion in section 3.2 when establishing this connection. In particular, a constrained version of the Michell truss design problem has also been proposed in 47] although not being completely equivalent to our formulation. However, they share the similar feature of avoiding infinitely large truss member sections (and are thus unable to sustain concentrated forces) but also of exhibiting a maximum load level.

\section{Numerical implementation and penalization procedure}

\subsection{Conic representation}

The fenics_optim package 11] enables to formulate convex variational problems provided that the involved convex functions admit a conic representation [10] i.e. can be expressed using linear equality/inequality constraints and conic constraints involving either the second-order Lorentz cone or the cone of semi-definite matrices. In particular, the package can be used to easily formulate and solve limit analysis problems 12. If $G$ can be formulated using second-order cone constraints, the associated problems (LOAD-MAX and (VOL-MIN will be SOCP problems. Similarly, if $G$ can be formulated using semi-definite constraints, problems (LOAD-MAX and (VOL-MIN will be Semi-Definite Programming (SDP) problems.

\subsection{Finite-element discretization}

For both problems (LOAD-MAX and (VOL-MIN $)$, the first two constraints related to the equilibrium condition and traction boundary conditions are in fact replaced by their weak form:

$$
\int_{\mathcal{D}} \boldsymbol{\sigma}: \nabla^{s} \boldsymbol{u} \mathrm{dx}=\int_{\mathcal{D}} \rho \boldsymbol{f} \cdot \boldsymbol{u} \mathrm{dx}+\int_{\partial \mathcal{D}_{T}} \lambda \boldsymbol{T} \cdot \boldsymbol{u}
$$

$\forall \boldsymbol{u}$ regular enough and satisfying the fixed displacement boundary conditions on $\partial \mathcal{D} \backslash \partial \mathcal{D}_{T}$ and where $\nabla^{s}$ is the symmetrized gradient operator. This weak form is discretized using discontinuous $\mathbb{P}^{1}$-Lagrange interpolation for $\boldsymbol{\sigma}$ and continuous $\mathbb{P}^{2}$-Lagrange interpolation for $\boldsymbol{u}$ and $\mathbb{P}^{1}$ for the density field $\rho$. The discretized version of the above equilibrium weak form would therefore read as:

$$
\mathbf{H} \boldsymbol{\Sigma}=\mathbf{F} \boldsymbol{\rho}+\lambda \mathbf{T}
$$

where $\boldsymbol{\Sigma}$ is the vector of stress unknowns and $\boldsymbol{\rho}$ the vector of density unknowns, $\boldsymbol{H}$ is the equilibrium matrix arising from the left-hand side of $(9), \mathbf{F}$ a matrix arising from the first right-hand side and $\mathbf{T}$ is the nodal force vectors corresponding to the boundary traction $\boldsymbol{T}$. 
The discrete LOAD-MAX problem therefore writes as:

$$
\begin{aligned}
\lambda^{+}=\max _{\lambda, \boldsymbol{\Sigma}, \boldsymbol{\rho}} & \lambda \\
\text { s.t. } & \mathbf{H} \boldsymbol{\Sigma}=\mathbf{F} \boldsymbol{\rho}+\lambda \mathbf{T} \quad \\
& \boldsymbol{\Sigma}^{i} \in \boldsymbol{\rho}^{i} G \\
& \boldsymbol{c}^{\mathrm{T}} \boldsymbol{\rho} \leq \eta \\
& 0 \leq \boldsymbol{\rho} \leq 1
\end{aligned}
$$

where $\boldsymbol{\Sigma}^{i}$ (resp. $\boldsymbol{\rho}^{i}$ ) denotes the value of the stress tensor $\boldsymbol{\sigma}$ (resp. pseudo-density $\rho$ ) at node $i$ and $\boldsymbol{c}$ is the vector obtained by discretizing the volume average operator such that $\frac{1}{|\mathcal{D}|} \int_{\mathcal{D}} \rho \mathrm{dx}=\boldsymbol{c}^{\mathrm{T}} \boldsymbol{\rho}$. Again, problem (11) involves only linear equality or inequality constraints except for the strength domain constraint $\boldsymbol{\Sigma}^{i} \in \boldsymbol{\rho}^{i} G$ which can be expressed using second-order cone constraints for the considered strength criteria.

Using the same notations, the discrete counterpart to (VOL-MIN reads as:

$$
\begin{aligned}
\eta^{-}=\min _{\boldsymbol{\Sigma}, \boldsymbol{\rho}} & \boldsymbol{c}^{\mathrm{T}} \boldsymbol{\rho} \\
\text { s.t. } & \mathbf{H} \boldsymbol{\Sigma}=\mathbf{F} \boldsymbol{\rho}+\lambda \mathbf{T} \\
& \boldsymbol{\Sigma}^{i} \in \boldsymbol{\rho}^{i} G \\
& 0 \leq \boldsymbol{\rho} \leq 1
\end{aligned} \quad \forall i
$$

For more details on the discrete formulation of the corresponding optimization problems, the reader can also refer to 22 .

\subsection{A penalization procedure}

As it will be seen in the next section, solution to problems (LOAD-MAX and (VOL-MIN produce diffuse density fields in general. In order to obtain a final truss-like design, some kind of penalization procedure must be used based on the initial continuous solution. Although it is not the main purpose of this paper, we shortly describe a possible strategy, which has never been done for plastic-based design to our knowledge.

We build upon the SIMP strategy by considering a penalized strength criterion of the form $\boldsymbol{\sigma} \in \rho^{p} G$ with $p \geq 1$. We will follow a continuation procedure starting from $p=1$ to $p=p_{\max }>1$ by solving a sequence of convex optimization problems. More precisely, at iteration $n$, we consider the following linearization of $\rho^{p_{n}}$ around the previous density solution $\rho_{n-1}$ :

$\rho^{p_{n}} \approx \rho_{n-1}^{p_{n}}+p_{n} \rho_{n-1}^{p_{n}-1}\left(\rho-\rho_{n-1}\right)=a_{n}+b_{n} \rho$

where $a_{n}=\left(1-p_{n}\right) \rho_{n-1}^{p_{n}}$ and $b_{n}=p_{n} \rho_{n-1}^{p_{n}-1}$. We therefore replace the penalized non-convex strength criterion constraint using the previous linear approximation as follows:

$\boldsymbol{\sigma} \in\left(a_{n}+b_{n} \rho\right) G$

Again, this constraint is convex and problems LOAD-MAX and VOL-MIN can be readily generalized using this constraint. Note that the original formulation is obtained for the particular case $p_{1}=1$, yielding $a_{1}=0$ and $b_{1}=1$. The update rule for the penalization exponent follows a heuristic which we found satisfying ${ }^{1}$. namely:

$$
p_{n+1}=1.1^{0.5+g_{n}} p_{n}
$$

where $g_{n}=\frac{4}{|\mathcal{D}|} \int_{\mathcal{D}} \rho_{n}\left(1-\rho_{n}\right) \mathrm{dx}$

with $g_{n}$ representing the average gray-level associated with $\rho_{n}$ or measure of non-discreteness as introduced in 44.

Finally, implementing only the above-mentioned procedure will lead to a well-known mesh dependency issue when $p>1$. This mesh-dependency is removed by adding a slope-control constraint 37] of the form:

$$
\|\nabla \rho\|_{2} \leq 1 / \ell
$$

where $\ell$ is a user-defined minimal characteristic length. Note again that this constraint is convex and directly fits into the second-order cone programming framework. It can be added at a small extra cost without impacting on the overall convergence of the interiorpoint algorithm. Note that we include this constraint only for the penalization phase when $p>1$.

\section{Illustrative applications}

\subsection{MBB beam}

\subsubsection{Initial convex solutions}

We first consider a MBB beam example (Figure 2) of length $l=36$ and height $h=6$ with simple supports on the left and roller supports on the right, a vertical force of reference intensity $P=1$ is applied at the top. In the following, only one half of the model will be represented, taking symmetry into account. Both supports and force are distributed over a small distance

1 This heuristic was inspired by one we found in http://www.cmap.polytechnique.fr/\%7Eallaire/map562/ console.simp.edp 


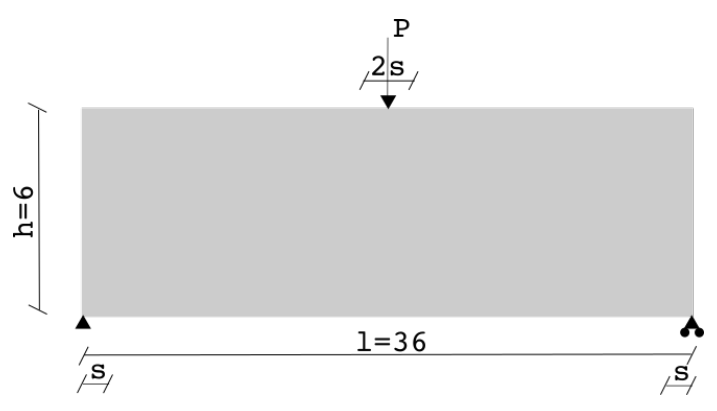

Fig. 2: A MBB beam example

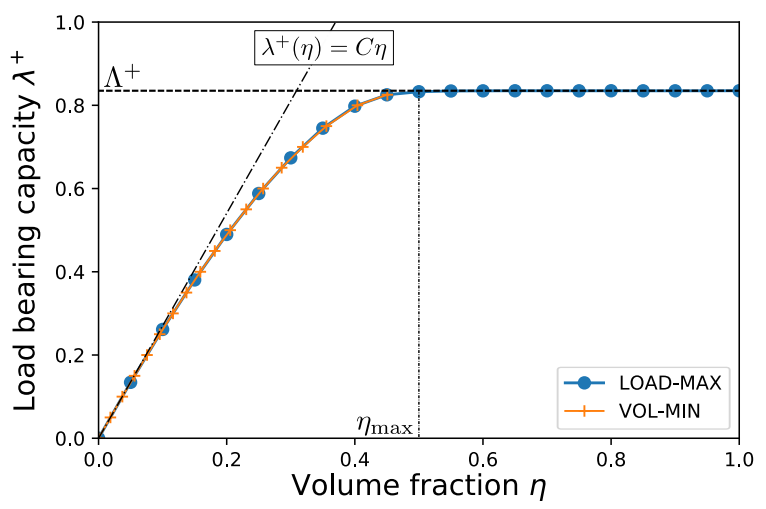

Fig. 3: Comparison between load maximization and volume minimization for the $L_{1}$-Rankine criterion. The horizontal line corresponds to the standard limit analysis solution $\Lambda^{+}$and the oblique line of equation $\lambda^{+}(\eta)=$ $C \eta$ to the solution of (LOAD-MAX) when removing the upper bound constraint $\rho \leq 1$.

$s=0.5$ to mitigate stress concentrations. The used finite-element mesh consists of approximately 40,000 elements.

We first consider the case of symmetric tensile/compressive strength $f_{c}=f_{t}=1$ using either a plane stress von Mises, Rankine or $L_{1}$-Rankine strength criterion. The load maximization problem LOAD-MAX is solved for increasing prescribed volume fractions $\eta$ while the volume minimization problem (VOL-MIN is solved for increasing imposed load factor $\lambda$. The results corresponding to the $L_{1}$-Rankine criterion are reported on Figure 3 .

As expected, both problems yield the same solution in terms of $\lambda^{+}(\eta)$. It should be noted that the volume minimization problem is only relevant for load factors less than the structure maximum load-bearing capacity $\Lambda^{+}$, the problem being unfeasible for larger imposed loads. On the contrary, the load maximization problem has a solution for any imposed volume fraction. The most interesting feature is the existence of a plateau where $\lambda^{+}(\eta)=\Lambda^{+}$for $\eta \geq \eta_{\max }=\eta^{-}\left(\Lambda^{+}\right)$. This can be explained by the fact that, for a given geometry, limit analysis solutions do not necessarily involve optimal stress fields lying at the boundary of the strength criterion everywhere in the domain. Some regions are indeed only weakly stressed or even unstressed so that a smaller strength criterion can be used. This leads to a material distribution with significantly lower total density while preserving the maximum load-bearing capacity. Interestingly, in the present example the material volume savings are significant since $\eta_{\max } \approx 50 \%$.

Solutions to the convex load maximization problems have been represented in Figure 4 for $\eta=20 \%$ for the three considered strength criteria. Obviously, the obtained fields do not exhibit a strong truss-like pattern which should be obtained through the use of a penalization procedure. This will be investigated in section 6.1 .2 . For now, let us investigate the convex solutions. First, roughly the same topologies are obtained for all criteria despite small differences, especially in the central diffuse region. Better insight can be gained when looking at the corresponding principal stresses (Figure 5). It can be seen that biaxial stress states are essentially present near the loading region, the supports or in the central region connecting the top compressed strut (in blue) and the bottom region in traction (in red). In order to assess more quantitatively which strength criterion yields stress fields close to being uniaxial, we computed throughout the domain the value of the following angle in the principal stress state:

$\theta=\arctan \left(\frac{\left|\sigma_{I I}\right|}{\left|\sigma_{I}\right|}\right)$

For uniaxial stress fields, either $\sigma_{I}$ or $\sigma_{I I}=0$ so that $\theta=0^{\circ}$ or $90^{\circ}$. Non-uniaxial stress fields will therefore correspond to values $0^{\circ}<\theta<90^{\circ}$. We therefore measure the amount of uniaxial stress fields in the obtained solution by representing the frequency distribution of $\theta$ for the three criteria. Figure 6a clearly indicates that the Rankine criterion produces much more biaxial stress states (near $45^{\circ}$ ) than the two others, which can well be explained by the criterion shape (see Figure 1a) which favours stress states lying on the square vertices. On this example, the difference between $L_{1}$-Rankine and von Mises criterion is not significant. However, if we change the way loading is applied by distributing it over a larger region $(s=5)$, one can clearly see in Figure 6b that the $L_{1}$-Rankine criterion is more efficient than the von Mises criterion at promoting uniaxial stress states. 


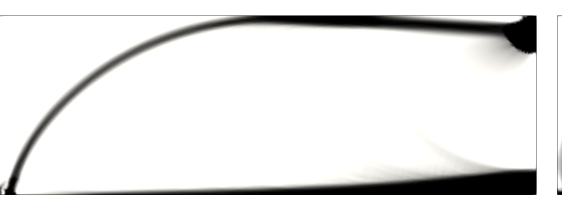

(a) $L_{1}$-Rankine criterion

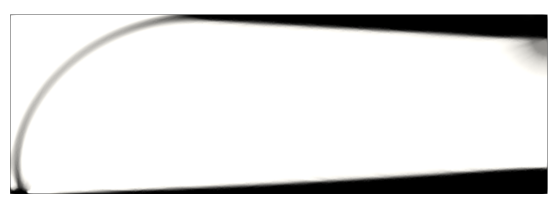

(b) Rankine criterion

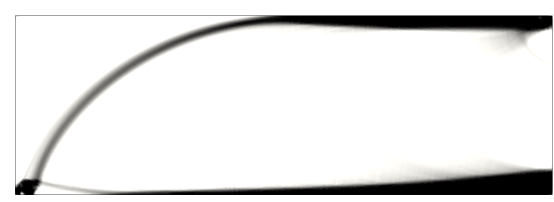

(c) von Mises criterion

Fig. 4: Optimized topology design of the MBB example using non-penalized load maximization problem for $\eta=0.20$

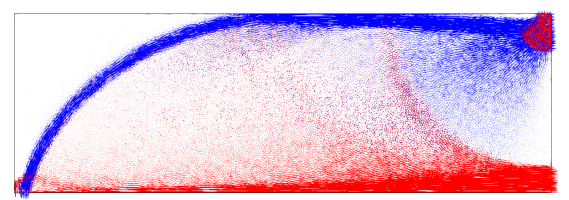

(a) $L_{1}$-Rankine criterion

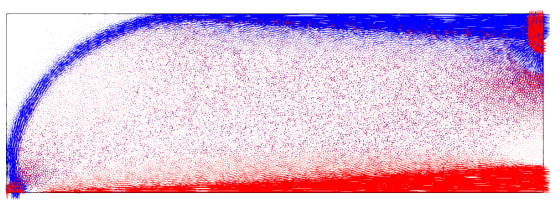

(b) Rankine criterion

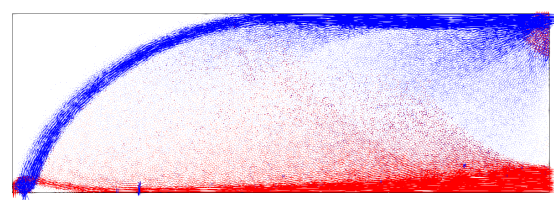

(c) von Mises criterion

Fig. 5: Principal stresses of the MBB example using non-penalized load maximization problem for $\eta=0.20$ (blue: compression, red:traction)

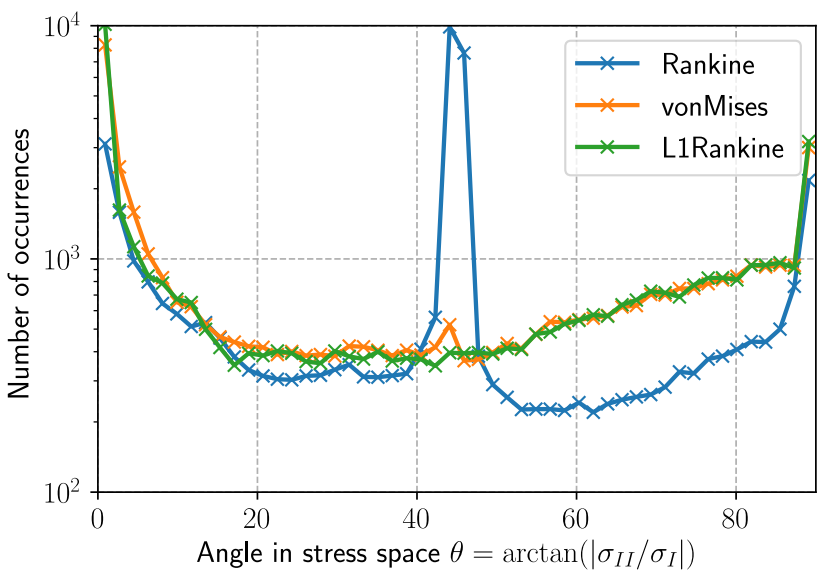

(a) With a concentrated load $(s=0.5)$

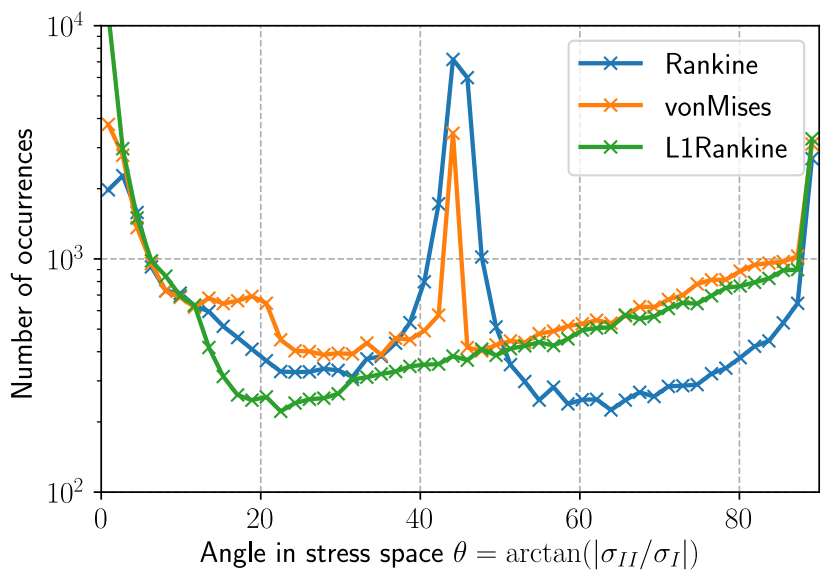

(b) With a more diffused load $(s=5$.)

Fig. 6: Frequency distribution of the $\theta$ angle for the MBB beam example with $\eta=0.2$

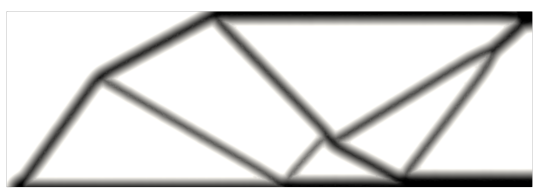

(a) $L_{1}$-Rankine (LOAD-MAX)

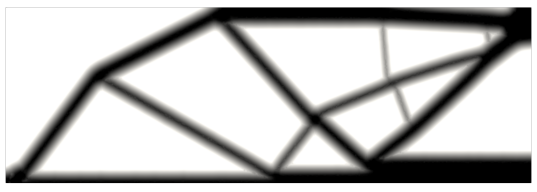

(d) $L_{1}$-Rankine VOL-MIN

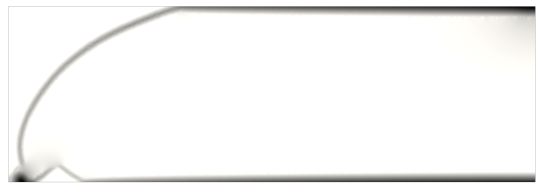

(b) Rankine (LOAD-MAX

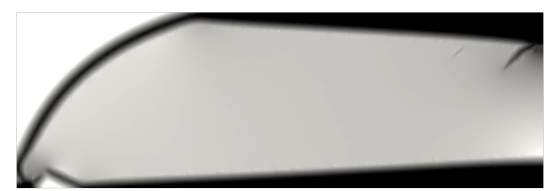

(e) Rankine VOL-MIN

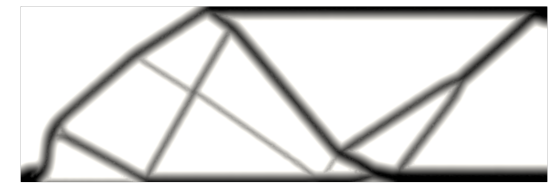

(c) von Mises LOAD-MAX

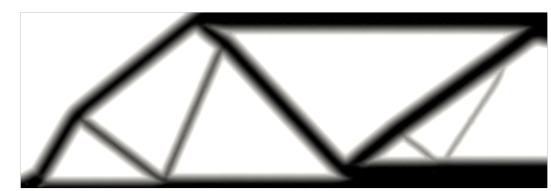

(f) von Mises VOL-MIN

Fig. 7: Optimized design of the MBB-beam using either penalized load maximization (top) or volume minimization (bottom) with different strength criteria 


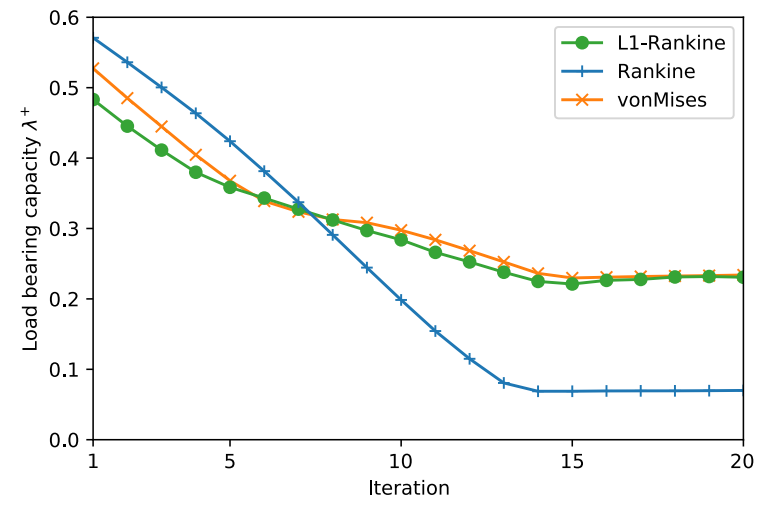

(a) Evolution of $\lambda^{+}$during penalization for LOAD-MAX

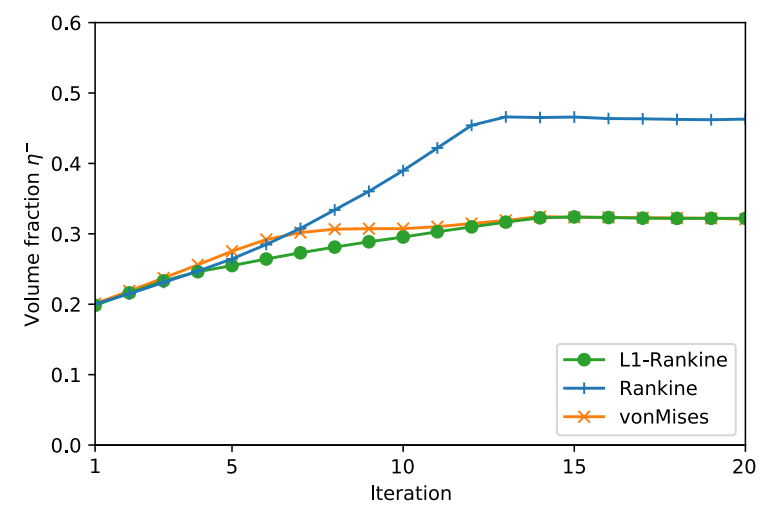

(b) Evolution of $\eta^{-}$during penalization for VOL-MIN

Fig. 8: Influence of the penalty procedure on the optimal objective functions for the (LOAD-MAX) and VOL-MIN problems

\subsubsection{Penalized solutions}

We now apply the penalization procedure described in section 5.3 for the three strength criteria. We use a slope control parameter $\ell=0.5$, set $p_{\max }=3$ and run the iterative procedure for 20 iterations which is usually enough to obtain a converged penalized design.

If the convex load maximization (LOAD-MAX and volume minimization (VOL-MIN) problems are equivalent for the initial solution, it will no longer be the case during the penalization procedure. Indeed, starting a penalizing sequence of (LOAD-MAX problems with $\eta=0.2$ will usually decrease the computed optimal load-bearing capacity $\lambda^{+}$. Similarly, starting a penalizing sequence of VOL-MIN problems with a loading corresponding to the initial $\lambda^{+}(\eta=0.2)$ will usually increase the computed optimal volume fraction $\eta^{-}$. The final penalized solutions are therefore not equivalent anymore since they correspond to different loadings and volume fractions. Figure 7 represents the obtained penalized design using either (LOAD-MAX) or VOL-MIN) for the three strength criteria. First, it can be observed that the penalization procedure systematically fails in the case of a Rankine strength criterion. This may be attributed to the fact that this strength criterion has a tendency to promote biaxial stress states over uniaxial stress states as already mentioned when discussing Figure 6. The penalization procedure is however efficient for the $L_{1}$-Rankine and von Mises for which truss-like designs are indeed obtained. The designs are quite different between both criteria, especially as regards members near the support and secondary members. Figure $8 \mathrm{a}$ (resp. 8b) shows the evolution of the computed loadbearing capacity (resp. volume fraction) during the different steps of the penalization procedure. As mentioned before, the penalization procedure tends to deteriorate the value of the objective function, it is however moderate for the $L_{1}$-Rankine and von Mises criteria, contrary to the Rankine criterion. Interestingly, the evolution of the objective function is very similar between the $L_{1}$-Rankine and von Mises criterion.

On Figure 9, we investigate the influence of the slope control parameter $\ell$ on the optimized solution. As expected, a small parameter yields a design with more members of shorter length. Increasing the value of this parameter results in optimized topologies with only a few members of larger length, their thickness is also spread out over a wide region, resulting in a gradient of intermediate densities. This is further confirmed by the final value of the measure of non-discreteness $g_{\text {end }}$ at the end of the penalization procedure which clearly increases for larger $\ell$. Conversely, the resulting load-bearing capacity $\lambda^{+}$decreases with a larger $\ell$, the resulting topology being less optimized for larger values of $\ell$. Finally, Figure 10 illustrates the influence of the imposed volume fraction $\eta$ on the final optimized topologies. As expected, the computed structures exhibit thicker members but also additional secondary members for larger values of $\eta$, resulting in a larger load-bearing capacity.

\subsection{Bridge example with asymmetric strengths}

The second example considers the design of a bridge structure (see Figure 11) with potentially asymmetric tensile/compressive strengths $f_{t}$ and $f_{c}$ using either the Rankine or $L_{1}$-Rankine criterion.

Figure 12 represents the evolution of the loadbearing capacity ratio as a function of $\eta$ for three different strength ratios: $f_{c}=\{0.1 ; 1 ; 10\}$ with $f_{t}=1$. One can observe that the obtained load-bearing 


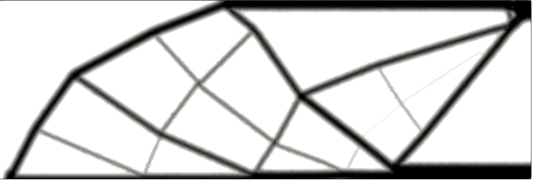

(a) $\ell=0.25: g_{\text {end }}=0.23, \lambda^{+}=0.297$

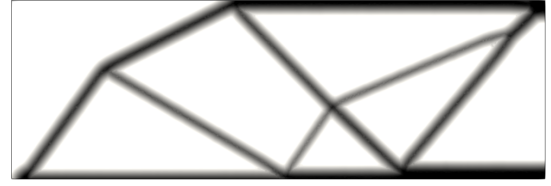

(b) $\ell=0.5: g_{\text {end }}=0.29, \lambda^{+}=0.237$

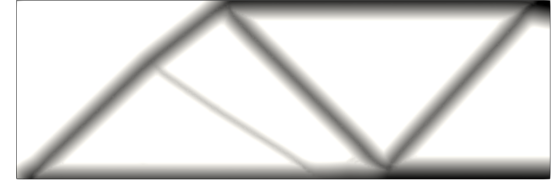

(c) $\ell=1: g_{\text {end }}=0.39, \lambda^{+}=0.154$

Fig. 9: Influence of the slope control parameter $\ell$ on the optimized topologies (LOAD-MAX with $\eta=0.2, L_{1}$-Rankine criterion)

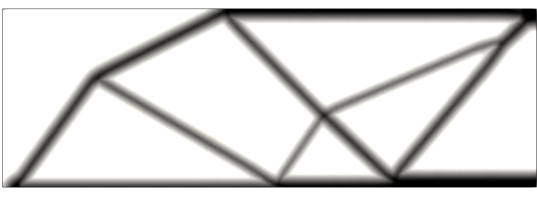

(a) $\eta=0.2: g_{\text {end }}=0.23, \lambda^{+}=0.297$

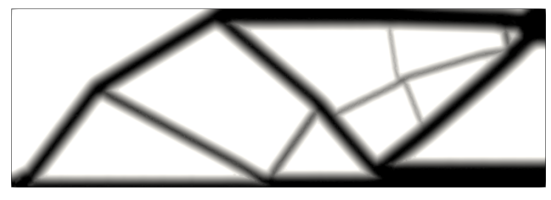

(b) $\eta=0.3: g_{\mathrm{end}}=0.30, \lambda^{+}=0.447$

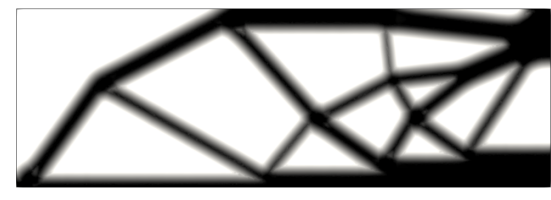

(c) $\eta=0.4: g_{\text {end }}=0.32, \lambda^{+}=0.628$

Fig. 10: Influence of the imposed volume fraction $\eta$ on the optimized topologies (LOAD-MAX with $\ell=0.5, L_{1}$-Rankine criterion)

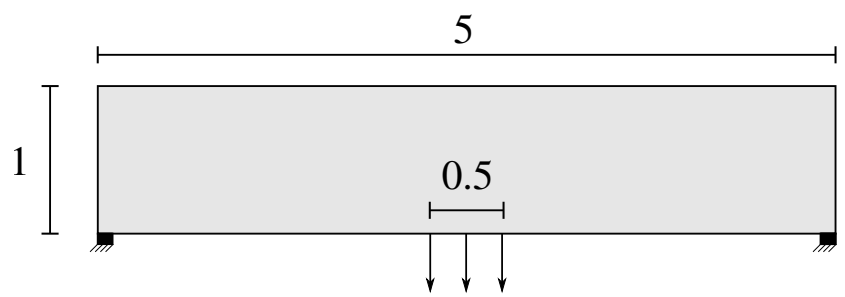

Fig. 11: A bridge structure with a central uniformly distributed loading $\boldsymbol{T}=-\boldsymbol{e}_{y}$. Fixed supports are distributed over regions of length 0.1 at both extremities.

capacities obtained with both criteria are quite similar for strongly asymmetric strengths whereas substantial differences are observed only for the symmetric case $f_{c}=f_{t}$. Although the stress domain corresponding to the $L_{1}$-Rankine criterion is much smaller than that of Rankine criterion, the load-bearing capacities are not so different for the asymmetric cases, indicating that the obtained solution are mostly uniaxial since both criteria coincide for uniaxial stress states. This is a further indication that the $L_{1}$-Rankine criterion can be used efficiently without being too conservative with respect to the predicted load-bearing capacity. Moreover, if analyzing the obtained truss-like structures with either the $L_{1}$-Rankine or the Rankine strength criterion, the difference in terms of limit load would be even less than that of Figure 12.

Figure 13 represents the obtained principal stress fields, either the initial unpenalized or the final pe-

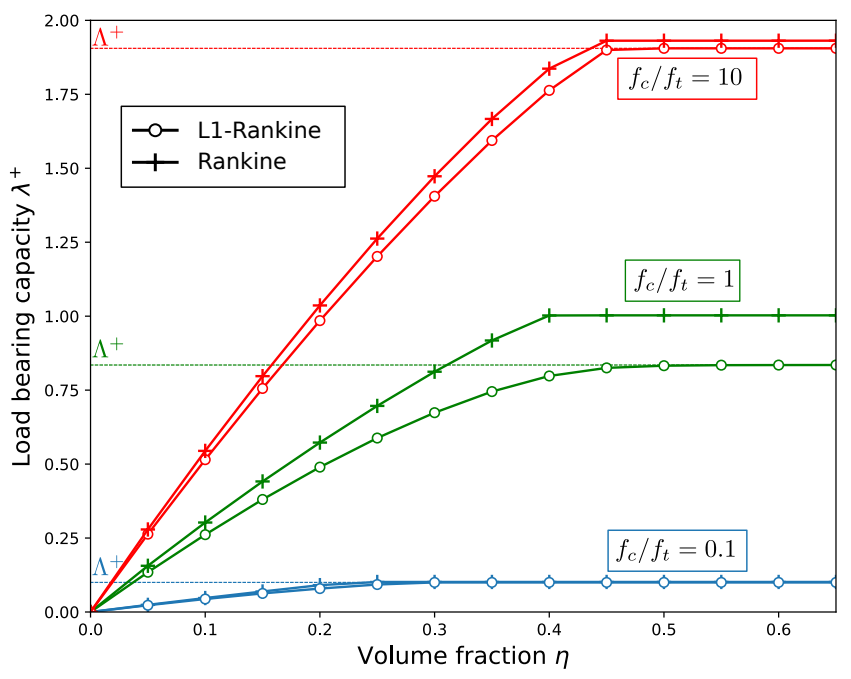

Fig. 12: Evolution of the load-bearing capacity for different strength asymmetry ratios

nalized solution, in the case of a larger compressive strength $f_{c}=10, f_{t}=1$ for the case $\eta=0.20$ with the $L_{1}$-Rankine criterion. Conversely, Figure 14 represents the corresponding solutions for the case of a smaller compressive strength $f_{c}=0.1$ and $f_{t}=1$. It can first be seen that the penalization procedure is very efficient even in these asymmetric cases and indeed produces truss-like patterns. The obtained patterns show a clearly distinct topology depending on the value of the compressive/tensile strength ratio. 


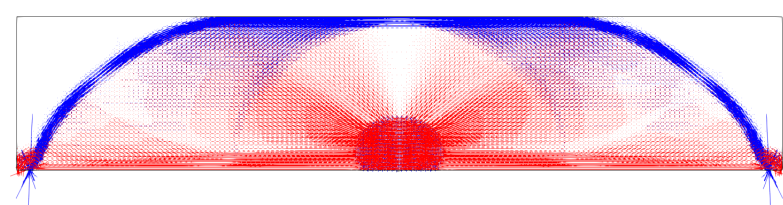

(a) Initial unpenalized solution

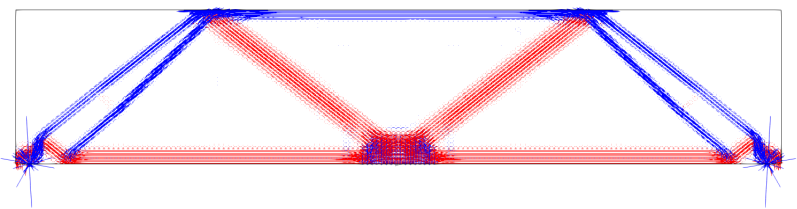

(b) Final penalized solution

Fig. 13: Principal stress distributions for the bridge example with $f_{t}=1$ and $f_{c}=10$ (blue: compression, red:traction)

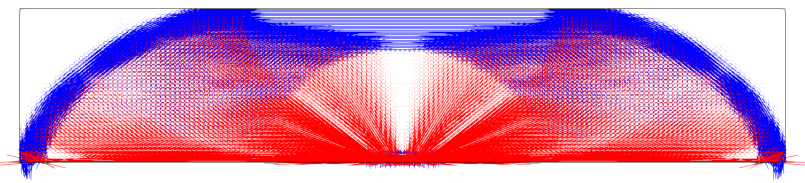

(a) Initial unpenalized solution

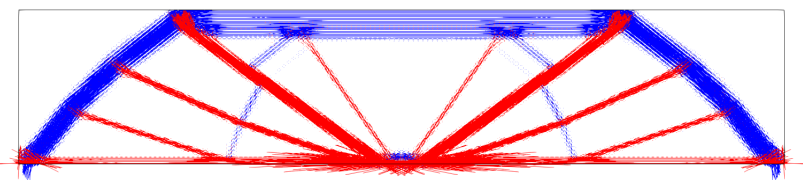

(b) Final penalized solution

Fig. 14: Principal stress distributions for the bridge example with $f_{t}=1$ and $f_{c}=0.1$ (blue: compression, red:traction)

\subsection{Example of a no-tension material}

We finish by illustrating the efficiency of our method on the important case of no-tension materials $f_{t}=0$ (in practice we still set some residual tension $f_{t}=10^{-3} f_{c}$ to avoid numerical issues). We investigate the case of the domain represented in Figure 15 subject to fixed supports on its bottom and lateral sides and uniformly distributed compressive $\boldsymbol{T}=-\boldsymbol{e}_{y}$ load on the top boundary. We investigate two different cases for the central region width with either $s=0$ or $s=0.3$. We set $\eta=0.2$ and $\ell=0.1$ for slope control.

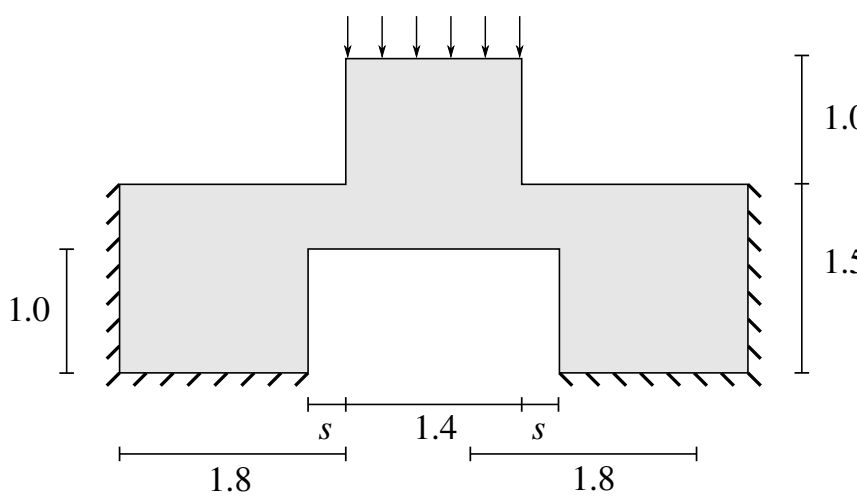

Fig. 15: Geometrical domain for optimization of a notension material

First, the principal stress distributions obtained from the resolution of the unpenalized convex problem for the case $s=0$ are represented in Figure 16a. We can remark that the obtained stress field is indeed in a purely compressive state with a localized uniaxial field in the inclined strut which is supported by the bottom boundary. The central top region is subject to a uniformly distributed uniaxial stress state. We can highlight that the obtained solution is therefore already extremely satisfying in terms of manufacturability. As a result, there is little use here for a penalization procedure, which will essentially replace the top uniform region by a series of columns and slightly modify the main struts inclination (Figure 16b).

Interestingly, when increasing the central opening width (case $s=0.3$ ). The previous solution now becomes impossible to sustain since the struts would have to kink to avoid the opening. Since this kink cannot be supported by a no-tension material, the final solution introduces a secondary strut which will be supported by the vertical boundaries in this case (Figure 17a). Again, the obtained solution is remarkably well localized and the penalization procedure only modifies the top uniform region (Figure 17b).

For the sake of comparison, we also used the Rankine criterion for the same examples. The different optimized pseudo-density fields are compared on Figures 19 and 20. Although being globally similar, especially concerning the number and inclination of the structure legs, both criteria yield different optimized topologies on the upper part of the domain serving to transmit the distributed loading to the supporting arch. In particular, the Rankine criterion produces a secondary arch and a horizontal member above the opening contrary to the $L_{1}$-Rankine criterion which results in an array of columns. This might suggest that, although members are in uniaxial stress states, connections between 


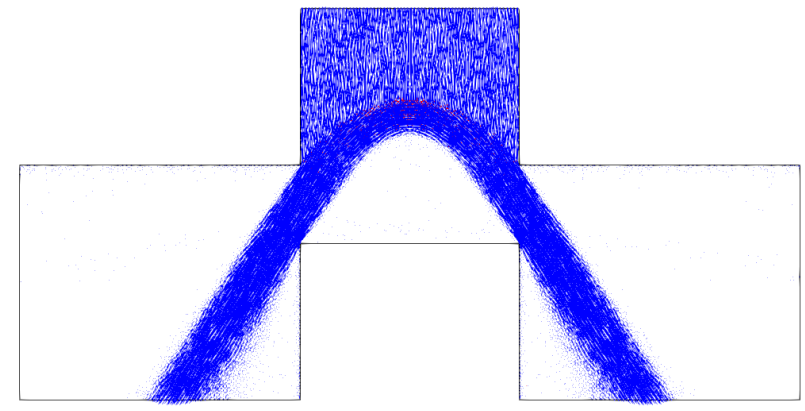

(a) Initial unpenalized solution

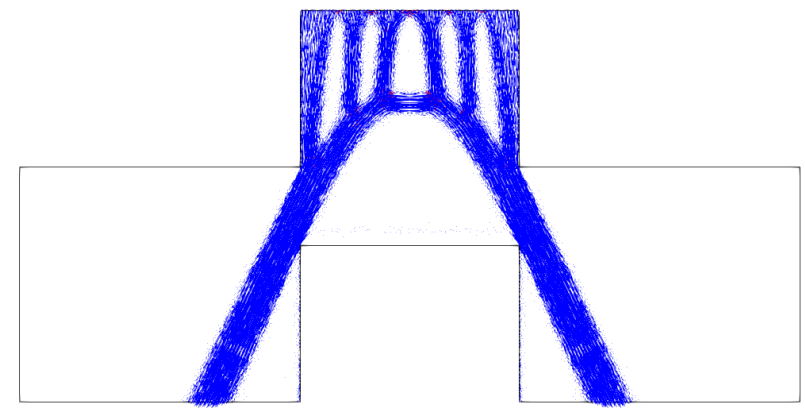

(b) Final penalized solution

Fig. 16: Principal stress distributions for the no-tension material example with $s=0$

members experience biaxial stress states which are influenced by the choice of the strength criterion and may impact the final optimized topologies. This aspect should deserve a more thorough investigation in future works.

Finally, the evolution of the load-bearing capacity obtained from the initial unpenalized solution as a function of the prescribed volume fraction $\eta$ has been represented in Figure 18 for both cases $s=0$ and $s=0.3$. Again, the curves follow the same trend as in Figure 3. Interestingly, the critical volume fraction for which the maximum load-bearing capacity $\Lambda^{+}$is attained is here $\eta_{\max } \approx 0.25-0.3$ for both situations, further showing that important material savings can be achieved through topology optimization without affecting the structural load-bearing capacity. Finally, although aesthetically pleasing, the obtained penalized solutions tend to decrease the final load-bearing capacity by roughly $20 \%$ (starred symbols in Figure 18).

\section{Conclusions}

This article aimed at proposing a general framework for unifying the concepts of topology optimization and limit analysis/yield design theory, which is frequently used in civil and structural engineering. Contrary to

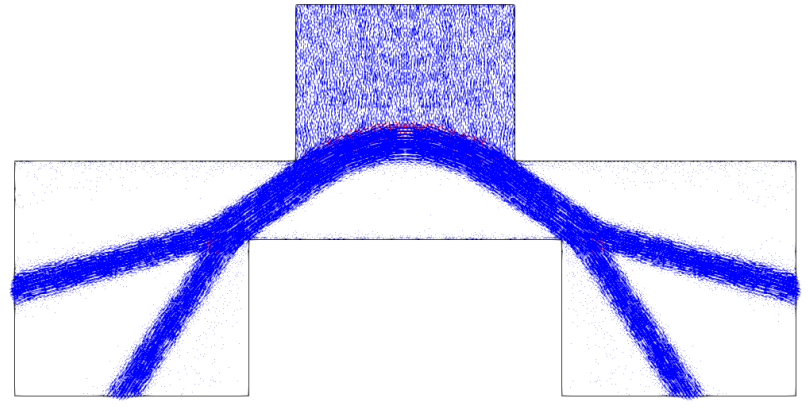

(a) Initial unpenalized solution

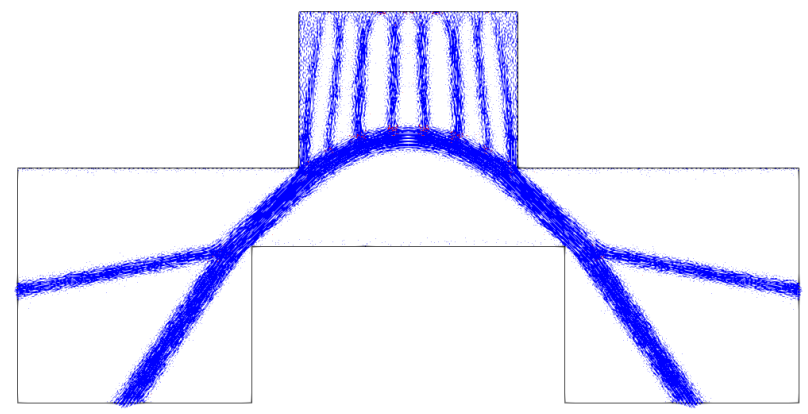

(b) Final penalized solution

Fig. 17: Principal stress distributions for the no-tension material example with $s=0.3$

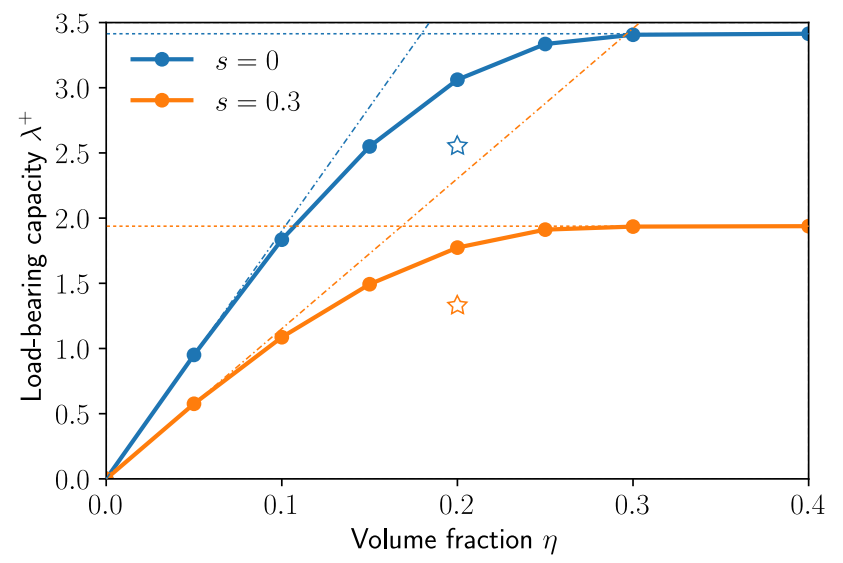

Fig. 18: Evolution of the initial load-bearing capacity for the no-tension material example as a function of the prescribed volume fraction. Starred symbols represent the load-bearing capacity obtained at the end of the penalization procedure. 


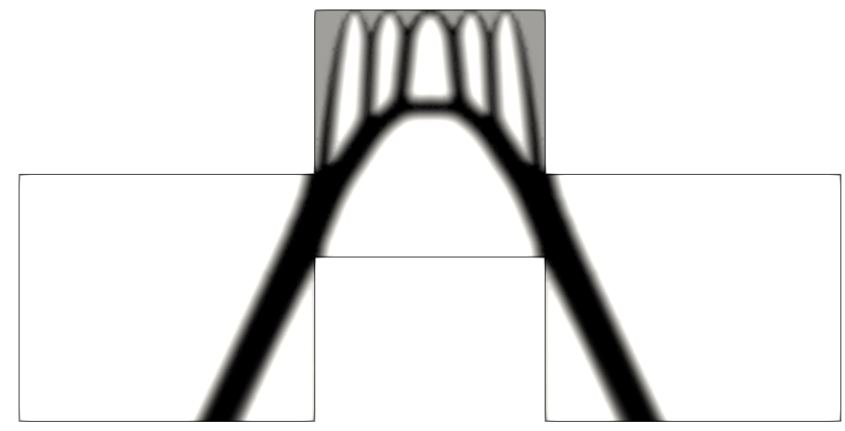

(a) $s=0$

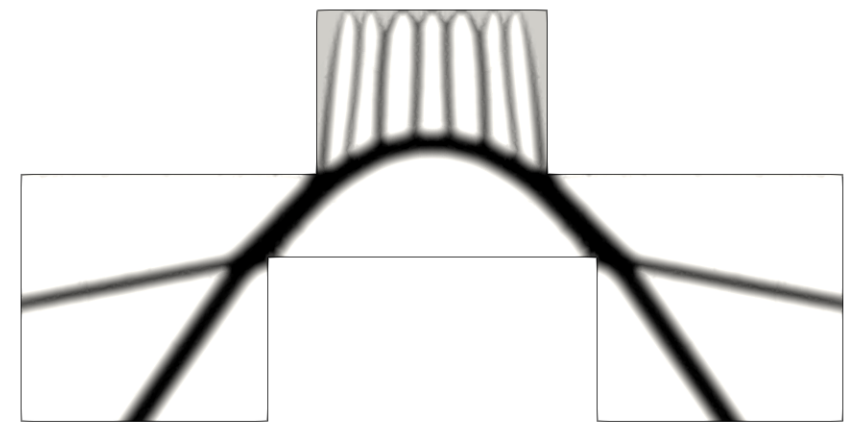

(b) $s=0.3$

Fig. 19: Optimized pseudo-density fields with the $L_{1^{-}}$ Rankine criterion

compliance-based topology optimization, geometry is here optimized with respect to the structure maximum load-bearing capacity for a fixed material volume fraction. An alternative approach consists in minimizing the total material volume under the constraint of supporting a fixed loading under the material local strength capacities. Both topology optimization problems are relaxed into convex problems, which can be cast as second-order programming problems for usual yield domains in 2D. The proposed optimization scheme allows optimizing for ultimate limit state (ULS) and can be used in combination with classical optimization for serviceability limit state (SLS). This is essential for civil engineering applications, where material with different compressive and tensile strengths are commonly used.

The main results of this work can be summarized as follows:

- both load maximization and volume minimization convex formulations are in fact two sides of the same coin, yielding the same solution;

- a small amount of material is often enough to support the load-bearing capacity obtained from classical limit analysis, offering important potential savings;

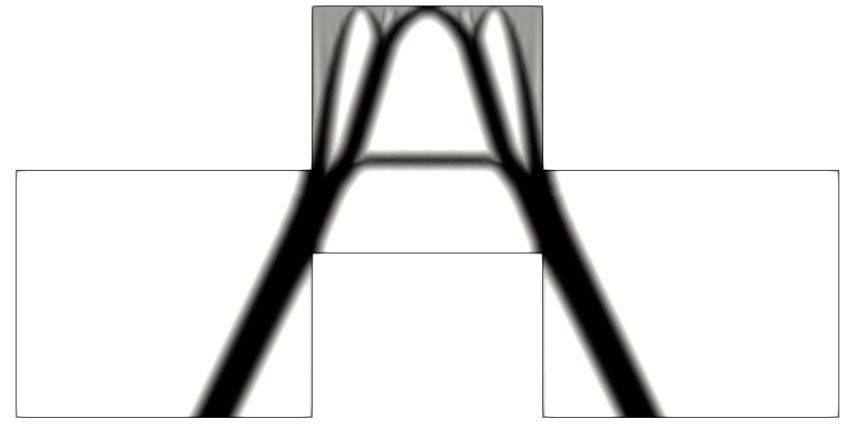

(a) $s=0$

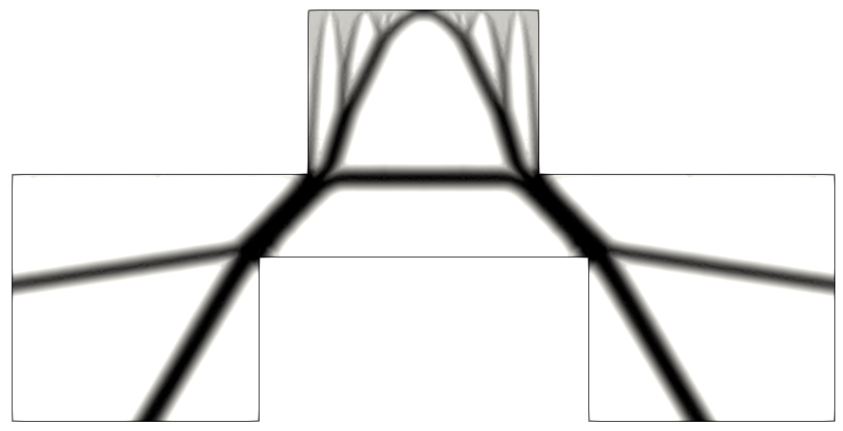

(b) $s=0.3$

Fig. 20: Optimized pseudo-density fields with the Rankine criterion

- different strength criteria can be used to model the underlying material properties. We propose using an original $L_{1}$-Rankine criterion which offers several advantages:

- it can model materials with different tensile/compressive strength, contrary to a von Mises criterion for instance;

- it provides a conceptual link with optimal truss theory 34 ;

- it tends to promote more efficiently uniaxial stress states, contrary to a more classical Rankine criterion for instance;

- no-tension materials can be handled without difficulty and, for such strongly asymmetric materials, the convex formulation tend to produce manufacturable truss-like designs;

- for other cases where the obtained solution is continuous, we propose an efficient penalization procedure based on an iterative resolution of such convex problems.

Interestingly, volume minimization with a stress constraint is generally different from the use of a stressdependent objective function with a volume constraint when dealing with elastic materials. This picture is clearly different in limit analysis-based formulations as 
we have shown. Although it can be proven mathematically, the fundamental origin of this equivalency is however still a bit elusive at this stage and should be investigated more deeply.

Future work will also be devoted to the generalization of the proposed formulation to bi-material optimization, e.g. reinforced concrete or fiber placement in composite laminates. Similarly to compliance-based optimization problems, the proposed framework should also be extended to the case involving multiple loadings as well as to the consideration of buckling constraints. Finally, in our considered numerical examples we did not consider any self-weight. The introduction of selfweight is known to introduce some numerical difficulties in SIMP-like compliance optimization as discussed in [16. This point should also deserve more attention in the future.

\section{A Proof that $\sigma \in \rho G$ is a convex constraint}

The constraint $\boldsymbol{\sigma} \in \rho G$ is equivalent to $\boldsymbol{\sigma} / \rho \in G$ for $\rho>0$. Let us consider any $\left(\boldsymbol{\sigma}_{1}, \rho_{1}\right)$ and $\left(\boldsymbol{\sigma}_{2}, \rho_{2}\right)$ such that $\rho_{i}>0$ and $\sigma_{i} / \rho_{i} \in G$ for $i=1,2$. Then, $\forall \lambda \in[0 ; 1]$ :

$$
\begin{aligned}
\frac{(1-\lambda) \boldsymbol{\sigma}_{1}+\lambda \boldsymbol{\sigma}_{2}}{(1-\lambda) \rho_{1}+\lambda \rho_{2}} & =\frac{(1-\lambda) \rho_{1}}{(1-\lambda) \rho_{1}+\lambda \rho_{2}} \frac{\boldsymbol{\sigma}_{1}}{\rho_{1}}+\frac{\lambda \rho_{2}}{(1-\lambda) \rho_{1}+\lambda \rho_{2}} \frac{\boldsymbol{\sigma}_{2}}{\rho_{2}} \\
& =(1-\mu) \frac{\boldsymbol{\sigma}_{1}}{\rho_{1}}+\mu \frac{\boldsymbol{\sigma}_{2}}{\rho_{2}}
\end{aligned}
$$

where $\mu=\frac{\lambda \rho_{2}}{(1-\lambda) \rho_{1}+\lambda \rho_{2}} \in[0 ; 1]$. Owing to the convexity of $G$, we obtain that $\frac{(1-\lambda) \sigma_{1}+\lambda \sigma_{2}}{(1-\lambda) \rho_{1}+\lambda \rho_{2}} \in G$ which proves that the set $\{(\boldsymbol{\sigma}, \rho)$ s.t. $\boldsymbol{\sigma} \in \rho G\}$ is convex.

\section{B Proofs of solution properties of the relaxed problems}

Property 1: $\forall \eta, \eta^{\prime} \in[0 ; 1]$ with $\eta \leq \eta^{\prime}$ and $\eta^{\prime}>0$ :

$\frac{\eta}{\eta^{\prime}} \lambda^{+}\left(\eta^{\prime}\right) \leq \lambda^{+}(\eta) \leq \lambda^{+}\left(\eta^{\prime}\right)$

Proof: Let $\boldsymbol{\sigma}$ and $\rho$ (resp. $\boldsymbol{\sigma}^{\prime}$ and $\rho^{\prime}$ ) the solutions to $\operatorname{LOAD-MAX}(\eta)$ (resp. to $\left.\operatorname{LOAD-MAX}\left(\eta^{\prime}\right)\right)$. Then, $\left(\lambda^{+}(\eta), \boldsymbol{\sigma}, \rho\right)$ is feasible for problem $\overline{\text { LOAD-MAX }}\left(\eta^{\prime}\right)$ so that $\lambda^{+}(\eta) \leq \lambda\left(\eta^{\prime}\right)$. Besides, let us define $\widehat{\lambda}=\frac{\eta}{\eta^{\prime}} \lambda^{+}\left(\eta^{\prime}\right), \widehat{\sigma}=\frac{\eta}{\eta^{\prime}} \sigma^{\prime}$ and $\widehat{\rho}=\frac{\eta}{\eta^{\prime}} \rho^{\prime}$. Then, since $0 \leq \frac{\eta}{\eta^{\prime}} \leq 1$, we have $0 \leq \widehat{\rho} \leq \rho^{\prime} \leq 1$ and $\frac{1}{|\mathcal{D}|} \int_{\mathcal{D}} \widehat{\rho} \mathrm{dx} \leq \frac{\eta}{\eta^{\prime}} \frac{1}{|\mathcal{D}|} \int_{\mathcal{D}} \rho^{\prime} \mathrm{dx}=\eta$. Moreover, $(\hat{\lambda}, \widehat{\boldsymbol{\sigma}}, \widehat{\rho})$ verify the equilibrium equations and we also have $\widehat{\sigma} \in \widehat{\rho} G$. Thus, we have a feasible point for problem $\operatorname{LOAD-MAX}(\eta)$ and one therefore has $\widehat{\lambda} \leq \lambda^{+}(\eta)$.

Property 2: $\forall \lambda, \lambda^{\prime} \in\left[0 ; \Lambda^{+}\right]$and $\lambda \leq \lambda^{\prime}$ and $\lambda^{\prime}>0$ :

$\eta^{-}(\lambda) \leq \frac{\lambda}{\lambda^{\prime}} \eta^{-}\left(\lambda^{\prime}\right) \leq \eta^{-}\left(\lambda^{\prime}\right)$
Proof: The proof follows the same ideas as Property 1 when defining $\left(\boldsymbol{\sigma}^{\prime}, \rho^{\prime}\right)$ solution to $\operatorname{VOL}-\mathrm{MIN}\left(\lambda^{\prime}\right)$ and $\widehat{\boldsymbol{\sigma}}=\frac{\lambda}{\lambda^{\prime}} \boldsymbol{\sigma}^{\prime}$ and $\hat{\rho}=\frac{\lambda}{\lambda^{\prime}} \rho^{\prime}$. We then easily show that it is a feasible point for $\frac{\lambda}{\operatorname{VOL}-\text { MIN }}(\lambda)$ associated with an objective value $\frac{1}{|\mathcal{D}|} \int_{\mathcal{D}} \widehat{\rho} \mathrm{dx}=\frac{\lambda}{\lambda^{\prime}} \eta^{-}\left(\lambda^{\prime}\right)$.

Property 3: $\lambda^{+}(\eta)$ and $\eta^{-}(\lambda)$ are non-decreasing functions. $\lambda^{+}(\eta)$ is continuous and $\eta^{-}(\lambda)$ is injective. Finally, we also have:

$\eta \Lambda^{+} \leq \lambda^{+}(\eta) \quad \forall \eta \in[0 ; 1]$

where $\Lambda^{+}=\lambda^{+}(1)$ is the ultimate load factor of the limit analysis problem.

Proof: The monotone property follows directly from Propositions 1 and 2 The continuity follows from Proposition 1 with $\lambda^{\prime}=\lambda+\epsilon$ and $\epsilon \rightarrow 0$. Injectivity of $\eta^{-}(\lambda)$ follows from Proposition 2 when assuming that $\eta^{-}(\lambda)=\eta^{-}\left(\lambda^{\prime}\right)$ then $\lambda=\lambda^{\prime}$. Finally, the inequality follows from the particular case $\eta^{\prime}=1$ in Proposition 1. Indeed, for problem (LOAD-MAX), one can take $\rho=1$ everywhere, yielding a classical limit analysis problem with an ultimate load factor $\Lambda^{+}=\lambda^{+}(1)$.

Property 4: We have:

$\eta^{-}\left(\lambda^{+}(\eta)\right) \leq \eta \quad \forall \eta \in[0 ; 1]$

$\lambda^{+}\left(\eta^{-}(\lambda)\right) \geq \lambda \quad \forall \lambda \in\left[0 ; \Lambda^{+}\right]$

Proof: Let $\left(\boldsymbol{\sigma}^{+}, \rho^{+}\right)$(resp. $\left.\left(\boldsymbol{\sigma}^{-}, \rho^{-}\right)\right)$be a solution to $(\operatorname{LOAD}-\operatorname{MAX})(\eta)$ (resp. $\operatorname{VOL-MIN}(\lambda))$. Then, $\left(\boldsymbol{\sigma}^{+}, \rho^{+}\right)$is a feasible point for $\operatorname{VOL-MIN}\left(\lambda^{+}(\eta)\right)$ associated with an objective value $\frac{1}{|\mathcal{D}|} \int_{\mathcal{D}} \widehat{\rho} \mathrm{dx}=\eta$. This proves the first inequality.

Similarly, $\left(\boldsymbol{\sigma}^{-}, \rho^{-}\right)$is a feasible point for $\operatorname{LOAD-MAX}\left(\eta^{-}(\lambda)\right)$ for a load factor $\lambda$. This proves the second inequality.

Property 5: For all $\lambda \in\left[0 ; \Lambda^{+}\right], \lambda^{+}\left(\eta^{-}(\lambda)\right)=\lambda$ i.e. $\lambda^{+}$is the (left) inverse of $\eta^{-}$meaning that both problems (VOL-MIN) and (LOAD-MAX) are in fact equivalent.

Proof: Let us write the first inequality of Proposition 4 for $\eta=\eta^{-}(\lambda)$ for $\lambda \in\left[0 ; \Lambda^{+}\right]$, then:

$\eta^{-}\left(\lambda^{+}\left(\eta^{-}(\lambda)\right)\right) \leq \eta^{-}(\lambda)$

However, from the second inequality of Proposition 4 $\lambda^{+}\left(\eta^{-}(\lambda)\right) \geq \lambda$. Using the fact that $\eta^{-}$is non-decreasing we also have that:

$\eta^{-}(\lambda) \leq \eta^{-}\left(\lambda^{+}\left(\eta^{-}(\lambda)\right)\right)$

Combining both results gives that $\eta^{-}\left(\lambda^{+}\left(\eta^{-}(\lambda)\right)\right)=\eta^{-}(\lambda)$ i.e. $\lambda^{+}\left(\eta^{-}(\lambda)\right)=\lambda$ due to $\eta^{-}$being injective. 


\section{Conic formulation for the $L_{1}$-Rankine criterion in $2 \mathrm{D}$}

We consider the following isotropic criterion for $2 \mathrm{D}$ stress tensors $\sigma$ :

$\boldsymbol{\sigma} \in G_{\text {L1-Rankine }} \Leftrightarrow g\left(\sigma_{I}\right)+g\left(\sigma_{I I}\right) \leq 1$

where $\sigma_{I, I I}$ are the principal stresses, $g(\sigma)=\max \left\{\frac{\sigma}{f_{t}} ;-\frac{\sigma}{f_{c}}\right\}$ and $f_{t}, f_{c}$ the tensile and compressive strengths respectively.

In $2 \mathrm{D}$, we explicitly have that:

$$
\begin{gathered}
\sigma_{I}=\frac{\sigma_{x x}+\sigma_{y y}}{2}+\frac{1}{2} \sqrt{\left(\sigma_{x x}-\sigma_{y y}\right)^{2}+4 \sigma_{x y}^{2}} \\
\sigma_{I I}=\frac{\sigma_{x x}+\sigma_{y y}}{2}-\frac{1}{2} \sqrt{\left(\sigma_{x x}-\sigma_{y y}\right)^{2}+4 \sigma_{x y}^{2}}
\end{gathered}
$$

Introducing $T=\sigma_{x x}+\sigma_{y y}$ and $R=\sqrt{\left(\sigma_{x x}-\sigma_{y y}\right)^{2}+4 \sigma_{x y}^{2}}$, we have that:

- If $\sigma_{I}, \sigma_{I I}>0$ :

$$
\sigma_{I}+\sigma_{I I} \leq f_{t} \Leftrightarrow T \leq f_{t}
$$

- If $\sigma_{I}, \sigma_{I I}<0$

$$
-\sigma_{I}-\sigma_{I I} \leq f_{c} \Leftrightarrow-T \leq f_{c}
$$

- If $\sigma_{I}>0$ and $\sigma_{I I}<0$ :

$$
\begin{aligned}
\frac{\sigma_{I}}{f_{t}}-\frac{\sigma_{I I}}{f_{c}} \leq 1 & \Leftrightarrow T\left(\frac{1}{2 f_{t}}-\frac{1}{2 f_{c}}\right)+R\left(\frac{1}{2 f_{t}}+\frac{1}{2 f_{c}}\right) \leq 1 \\
& \Leftrightarrow R \leq\left(\frac{2 f_{t} f_{c}}{f_{c}+f_{t}}-T \frac{f_{c}-f_{t}}{f_{c}+f_{t}}\right)
\end{aligned}
$$

Denoting by $\alpha=\frac{f_{c}-f_{t}}{f_{c}+f_{t}}$ and $\bar{f}=\frac{2 f_{t} f_{c}}{f_{c}+f_{t}}$, we finally have the following conic formulation:

$$
\begin{aligned}
\sigma \in G_{\text {L1-Rankine }} \Leftrightarrow & -f_{c} \leq \sigma_{x x}+\sigma_{y y} \leq f_{t} \\
& {\left[\begin{array}{ccc}
\alpha & \alpha & 0 \\
1 & -1 & 0 \\
0 & 0 & 2
\end{array}\right]\left\{\begin{array}{l}
\sigma_{x x} \\
\sigma_{y y} \\
\sigma_{x y}
\end{array}\right\}+\left\{\begin{array}{l}
X_{0} \\
X_{1} \\
X_{2}
\end{array}\right\}=\left\{\begin{array}{l}
\bar{f} \\
0 \\
0
\end{array}\right\} } \\
& X_{0} \geq \sqrt{X_{1}^{2}+X_{2}^{2}}
\end{aligned}
$$

where the last condition is a quadratic conic constraint. have:

Finally, in the case of a density dependent criterion, we

$$
\begin{aligned}
\boldsymbol{\sigma} \in \rho G_{\text {L1-Rankine }} \Leftrightarrow & -\rho f_{c} \leq \sigma_{x x}+\sigma_{y y} \leq \rho f_{t} \\
& {\left[\begin{array}{ccc}
\alpha & \alpha & 0 \\
1 & -1 & 0 \\
0 & 0 & 2
\end{array}\right]\left\{\begin{array}{l}
\sigma_{x x} \\
\sigma_{y y} \\
\sigma_{x y}
\end{array}\right\}+\left\{\begin{array}{l}
X_{0} \\
X_{1} \\
X_{2}
\end{array}\right\}=\left\{\begin{array}{c}
\rho \bar{f} \\
0 \\
0
\end{array}\right\} }
\end{aligned}
$$

$$
X_{0} \geq \sqrt{X_{1}^{2}+X_{2}^{2}}
$$

\section{Conflict of interest}

The authors declare that they have no conflict of interest.

\section{Replication of results}

The Python code for implementing the topology optimization and for reproducing the manuscript examples is available as a supplementary material. This code relies on the fenics_optim Python package [11, itself relying on the FEniCS finite-element software library https://fenicsproject. org/ and the Mosek conic optimization solver https://www . mosek.com/

\section{References}

1. Allaire, G.: The homogenization method for topology and shape optimization. In: Topology optimization in structural mechanics, pp. 101-133. Springer (1997)

2. Allaire, G.: Shape optimization by the homogenization method, vol. 146. Springer Science \& Business Media (2012)

3. Allaire, G., Jouve, F., Toader, A.M.: Structural optimization using sensitivity analysis and a level-set method. Journal of computational physics 194(1), 363-393 (2004)

4. Amir, O.: Stress-constrained continuum topology optimization: a new approach based on elasto-plasticity. Structural and Multidisciplinary Optimization 55(5), 1797-1818 (2017)

5. Amir, O., Bogomolny, M.: Conceptual design of reinforced concrete structures using topology optimization with elastoplastic material modeling. International Journal for Numerical Methods in Engineering 90, 1578-1597 (2012). DOI 10.1002/nme.4253

6. Bach, F., Jenatton, R., Mairal, J., Obozinski, G., et al.: Convex optimization with sparsity-inducing norms. Optimization for Machine Learning 5, 19-53 (2011)

7. Bendsøe, M.P., Kikuchi, N.: Generating optimal topologies in structural design using a homogenization method. Computer Methods in Applied Mechanics and Engineering $\mathbf{7 1}(2), 197-224$ (1988). DOI https://doi.org/10.1016/0045-7825(88)90086-2. URL http://www.sciencedirect.com/science/article/pii/ 0045782588900862

8. Bendsøe, M.P., Sigmund, O.: Topology optimization: theory, methods, and applications, 2. ed., corr. printing edn. Engineering online library. Springer (2004). OCLC: 249399186

9. Bisbos, C., Pardalos, P.: Second-order cone and semidefinite representations of material failure criteria. Journal of Optimization Theory and Applications 134(2), 275-301 (2007)

10. Bleyer, J.: Automating the formulation and resolution of convex variational problems: Applications from image processing to computational mechanics. ACM Trans. Math. Softw. O(ja) (2020). DOI 10.1145/3393881. URL https://doi.org/10.1145/3393881

11. Bleyer, J.: fenics-optim - Convex optimization interface in FEniCS (2020). DOI 10.5281/zenodo.3604086. URL https://doi.org/10.5281/zenodo.3604086

12. Bleyer, J., Hassen, G.: Automated formulation and resolution of limit analysis problems. arXiv preprint arXiv:2005.04779 (2020) 
13. Block, P., Ochsendorf, J.: Thrust network analysis: A new methodology for three-dimensional equilibrium. Journal of the International Association for Shell and Spatial Structures 48 (2007)

14. Block, P., Ochsendorf, J.: Lower-bound analysis of masonry vaults, pp. 613-620. CRC Press (2008). DOI 10.1201/9781439828229.ch67

15. Block, P., Rippmann, M., Van Mele, T.: Compressive assemblies: Bottom-up performance for a new form of construction. AD Architectural Design 87(4), 104-109 (2017). DOI 10.1002/ad.2202. Special issue S. Tibbits (Ed.) - Autonomous Assembly: Designing for a new era of collective construction

16. Bruyneel, M., Duysinx, P.: Note on topology optimization of continuum structures including self-weight. Structural and Multidisciplinary Optimization 29(4), 245-256 (2005)

17. Chen, W.F.: Plasticity in reinforced concrete. J. Ross Publishing (2007)

18. Chen, W.F.: Limit analysis and soil plasticity. Elsevier (2013)

19. Damkilde, L., Krenk, S.: Limits-a system for limit state analysis and optimal material layout. Computers \& structures 64(1-4), 709-718 (1997)

20. Duysinx, P., Bendsøe, M.P.: Topology optimization of continuum structures with local stress constraints. International journal for numerical methods in engineering 43(8), 1453-1478 (1998)

21. Fin, J., Borges, L., Fancello, E.: Structural topology optimization under limit analysis. Structural and Multidisciplinary Optimization 59 (2018). DOI 10.1007/ s00158-018-2132-y

22. Herfelt, M., Poulsen, P., Hoang, L.: Strength-based topology optimisation of plastic isotropic von mises materials. Structural and Multidisciplinary Optimization (2018). DOI 10.1007/s00158-018-2108-y

23. Heyman, J.: The Stone Skeleton: Structural Engineering of Masonry Architecture. Cambridge University Press (1995). DOI 10.1017/CBO9781107050310

24. Heyman, J.: Arches, vaults, and buttresses: masonry structures and their engineering. Collected studies. Variorum (1996). URL https://books.google.fr/books? id=niNSAAAAMAAJ

25. Hill, R.: The mathematical theory of plasticity. Clarendon Press, Oxford (1950)

26. Horvath, A.: Construction materials and the environment. Annu. Rev. Environ. Resour. 29, 181-204 (2004)

27. Kammoun, Z., Smaoui, H.: A direct approach for continuous topology optimization subject to admissible loading. Comptes Rendus Mécanique 342(9), 520-531 (2014)

28. Kilian, M., Pellis, D., Wallner, J., Pottmann, H.: Material-minimizing forms and structures. ACM Transactions on Graphics (TOG) 36(6), 173 (2017)

29. Krabbenhøft, K., Lyamin, A.V., Sloan, S.W.: Threedimensional mohr-coulomb limit analysis using semidefinite programming. Communications in Numerical Methods in Engineering 24(11), 1107-1119 (2008)

30. Lobo, M.S., Vandenberghe, L., Boyd, S., Lebret, H.: Applications of second-order cone programming. Linear algebra and its applications 284(1-3), 193-228 (1998)

31. Makrodimopoulos, A., Martin, C.: Upper bound limit analysis using simplex strain elements and second-order cone programming. International journal for numerical and analytical methods in geomechanics 31(6), 835-865 (2007)
32. Maute, K., Schwarz, S., Ramm, E.: Adaptive topology optimization of elastoplastic structures 15(2), 81-91 (1998). DOI 10.1007/BF01278493. URL http://link. springer.com/10.1007/BF01278493

33. Mele, T.V., Mehrotra, A., Echenagucia, M.T., Frick, U., Ochsendorf, J., Dejong, M., Block, P.: Form finding and structural analysis of a freeform stone vault. In: Proceedings of IASS Annual Symposia, 8, pp. 1-10. International Association for Shell and Spatial Structures (IASS) (2016)

34. Michell, A.G.M.: LVIII. The limits of economy of material in frame-structures. The London, Edinburgh, and Dublin Philosophical Magazine and Journal of Science 8(47), 589-597 (1904)

35. Nielsen, M.P., Hoang, L.C.: Limit analysis and concrete plasticity. CRC press (2016)

36. Pedersen, P.: Some general optimal design results using anisotropic, power law nonlinear elasticity. Structural Optimization 15(2), 73-80 (1998). DOI 10. 1007/bf01278492. URL https://link.springer.com/ article/10.1007/BF01278492

37. Petersson, J., Sigmund, O.: Slope constrained topology optimization. International Journal for Numerical Methods in Engineering 41(8), 1417-1434 (1998)

38. Prager, W.: A note on discretized michell structures. Computer Methods in Applied Mechanics and Engineering 3(3), $349-355$ (1974). DOI https://doi.org/10.1016/0045-7825(74)90019-X. URL http://www.sciencedirect.com/science/article/pii/ 004578257490019X

39. Prager, W., Shield, R.T.: A general theory of optimal plastic design. Journal of Applied Mechanics, Transactions ASME 34(1), 184-186 (1964). DOI 10.1115/1. 3607621

40. Salençon, J.: Calcul à la rupture et analyse limite. Presses de l'Ecole Nationale des Ponts et Chaussées (1983)

41. Salençon, J.: Yield Design. London, Hoboken : ISTE Ltd., John Wiley \& Sons, Inc. (2013)

42. Schlaich, J., Schafer, K., Jennewein, M.: Toward a consistent design of structural concrete. PCI Journal 32(3), 74-150 (1987). DOI 10.15554/pcij.05011987.74.150. URL http://www.pci.org/pci_journal-1987-may-june-5/

43. Sigmund, O.: A 99 line topology optimization code written in Matlab. Structural and Multidisciplinary Optimization 21(2), 120-127 (2001). DOI 10.1007/ s001580050176. URL http://link.springer.com/10. $1007 / \mathrm{s} 001580050176$

44. Sigmund, O.: Morphology-based black and white filters for topology optimization. Structural and Multidisciplinary Optimization 33(4-5), 401-424 (2007)

45. Sigmund, O., Maute, K.: Topology optimization approaches. Structural and Multidisciplinary Optimization 48(6), 1031-1055 (2013). DOI 10.1007/ s00158-013-0978-6. URL https://doi.org/10.1007/ s00158-013-0978-6

46. Sloan, S.W.: Lower bound limit analysis using finite elements and linear programming. International Journal for Numerical and Analytical Methods in Geomechanics 12(1), 61-77 (1988)

47. Strang, G., Kohn, R.V.: Hencky-Prandtl nets and constrained Michell trusses. Computer Methods in Applied Mechanics and Engineering 36(2), 207-222 (1983)

48. Svanberg, K.: The method of moving asymptotes - a new method for structural optimization. International Journal for Numerical Methods in Engineering 24(2), 359-373 (1987). DOI 10.1002/nme. 
1620240207. URL https://onlinelibrary.wiley.com/ doi/abs/10.1002/nme.1620240207

49. Swan, C.C., Kosaka, I.: Voigt-reuss topology optimization for structures with nonlinear material behaviors. International Journal for Numerical Methods in Engineering 40(20), 3785-3814 (1997). DOI 10.1002/(SICI)1097-0207(19971030)40:20<3785:: AID-NME240/3.0.CO;2-V. URL http://doi.wiley. com $/ 10.1002 / \% 28$ SICI $\% 291097-0207 \% 2819971030 \% 2940 \%$ 3A20\%3C3785\%3A\%3AAID-NME240\%3E3.0.CO\%3B2-V

50. Vincent, H., Arquier, M., Bleyer, J., De Buhan, P.: Yield design-based numerical analysis of three-dimensional reinforced concrete structures. International Journal for Numerical and Analytical Methods in Geomechanics 42(18), 2177-2192 (2018). DOI 10.1002/nag. 2850. URL https://hal-enpc.archives-ouvertes.fr/ hal-01855342

51. Wallin, M., Jönsson, V., Wingren, E.: Topology optimization based on finite strain plasticity. Structural and multidisciplinary optimization 54(4), 783-793 (2016) 